\title{
Supernovae Producing Unbound Binaries and Triples
}

\author{
C. S. Kochanek ${ }^{1,2}$ \\ 1 Department of Astronomy, The Ohio State University, 140 West 18th Avenue, Columbus OH 43210 \\ ${ }^{2}$ Center for Cosmology and AstroParticle Physics, The Ohio State University, $191 \mathrm{~W}$. Woodruff Avenue, Columbus OH 43210
}

' 30 July 2021

\begin{abstract}
The fraction of stars which are in binaries or triples at the time of stellar death and the fraction of these systems which survive the supernova (SN) explosion are crucial constraints for evolution models and predictions for gravitational wave source populations. These fractions are also subject to direct observational determination. Here we search 10 supernova remnants (SNR) containing compact objects with proper motions for unbound binaries or triples using Gaia EDR3 and new statistical methods and tests for false positives. We confirm the one known example of an unbound binary, HD 37424 in G180.0-01.7, and find no other examples. Combining this with our previous searches for bound and unbound binaries, and assuming no bias in favor of finding interacting binaries, we find that $72.0 \%(52.2 \%-86.4 \%, 90 \%$ confidence) of SN producing neutron stars are not binaries at the time of explosion, $13.9 \%(5.4 \%-27.2 \%)$ produce bound binaries and $12.5 \%(2.8 \%-31.3 \%)$ produce unbound binaries. With a strong bias in favor of finding interacting binaries, the medians shift to $76.0 \%$ were not binaries at death, $9.5 \%$ leave bound and $13.2 \%$ leave unbound binaries. Of explosions that do not leave binaries, $<18.9 \%$ can be fully unbound triples. These limits are conservatively for $M>5 M_{\odot}$ stars, although the mass limits for individual systems are significantly stronger. At birth, the progenitor of PSR J0538+2817 was probably a $13-19 M_{\odot}$ star, and at the time of explosion it was probably a Roche limited, partially stripped star transferring mass to HD 37424 and then producing a Type IIL or IIb supernova.
\end{abstract}

Key words: stars: massive - supernovae: general - supernovae

\section{INTRODUCTION}

Massive stars are not isolated. Almost all start in binaries (e.g., Kobulnicky \& Frver 2007, Sana et al. 2012, Moe \& Di Stefano 2017, Villaseñor et al. 2021) and many , are in triple or still higher order systems (Moe \& Di Stefano 2017). The evolution of these systems is crucial to understanding the expected properties of supernovae $(\mathrm{SN})$ and compact objects and there is a long history of using binary population synthesis (BPS) models to try and follow the evolution of binary populations (e.g., Belczvnski et al. 2008, Eldridge et al. 2017). These population studies have recently expanded to follow the evolution of triple or quadruple systems with their far richer dynamical properties (e.g., Toonen et al. 2020, Hamers et al. 2021). The end results for the evolution of massive star binaries and triples are particularly crucial to understanding the growing sample of compact object mergers found through gravitational wave emission (e.g., Abbott et al. 2021). \begin{tabular}{|crrrr}
\multicolumn{1}{c|}{ While } & studies & like & Kobulnicky \& Frver & \\
Sana et al. & $(2012)$, & Moe \& Di Stefano & $(2017)$ & and
\end{tabular}
Villaseñor et al. (2021) are excellent surveys of the multiplicity of massive stars, the path to mergers or interacting compact objects is long and complex. In addition to standard problems in stellar evolution, there are the added problems of mass transfer, common envelope evolution, neutron star (or black hole) birth kicks (see, e.g., the review of BPS models by Han et al. 2020) and the nature of the mapping between core collapse and outcome (supernova or failed supernovae, neutrons star or black hole etc., see, e.g., Pejcha \& Thompson 2015, Kochanek 2014, Sukhbold et al. 2016). All of the physical effects are both uncertain and important for any prediction, including for the population of merging compact objects.

This process would be better constrained if we understood the multiplicity of systems containing compact objects and the fraction of initially multiple systems that survive the death of a member star. Compact object mergers, a small minority of the initial systems lying at the very end a complex evolutionary history, are likely a very poor way to understand this problem. Similarly, interacting neutron star and black hole binaries are a small. biased fraction of bina- 
ries containing compact objects. Searches for non-interacting neutron star (other than pulsars) and black hole binaries are difficult and only just starting (e.g., Thompson et al. 2019, Javasinghe et al. 2021) and searches for unbound compact objects only become fully practical as part of the microlensing component of the Roman Space Telescope (e.g., Lam et al. 2020).

There is, however, a second point in the evolution of these systems where it is possible to make progress. Studies of supernovae remnants (SNRs) can be used to determine the multiplicity of massive stars just before and after the death of a member. This was originally conceived of as a search for O/B stars associated with SNRs, potentially with peculiar kinematics (van den Bergh 1980, Guseinov et al. 2005). However, current models (e.g., Renzo et al. 2019), and data (e.g., Kochanek et al. 2019) suggest that the kinematics of these stars are not very distinctive. Nonetheless, given an estimate of the explosion site based on the centroid or expansion of the SNR, one can try to identify stars consistent with that location at the estimated time of explosion.

The easiest systems to examine are the very youngest SNRs: the Crab, Cas A and SN 1987A. Because of their youth, the region that must be searched even for unbound companions is so small that it is trivial to rule out bound or unbound companions down to mass limits of $\sim M_{\odot}$ or less (Kochanek 2018, also see Kerzendorf et al. 2019 and Fraser \& Boubert 2019). The lack of a companion in the Type IIb (Krause et al. 2008, Rest et al. 2011) SN Cas A is particularly interesting because models for producing Type IIb SNe typically rely on mass transfer (e.g., Benvenuto et al. 2013). The lack of a companion to SN 1987A is consistent with models of the progenitor as a merger remnant (e.g., Podsiadlowski 1992).

Older SNRs with identified compact remnants and limited extinction are easy to search for bound remnants simply using the properties of the optical counterpart to the neutron star. Kochanek et al. (2019) identified 23 Galactic SNRs where it was practical to carry out such a search, finding no non-interacting binaries and three previously identified interacting binaries. One of these systems, SS 433, is a Roche accreting system where the compact object is generally believed to be a black hole (see, e.g., Hillwig \& Gies 2008). The other two systems, HESS J0632+057 (Hinton et al. 2009) and 1FGL J1018.6-5856 (Corbet et al. 2011) are neutron star, wind accretion, high mass X-ray binaries (HMXBs). Kochanek et al. (2019) speculated that shock heating of the companion by the SN blast wave might drive it to have stronger winds for a period of time after the SN explosion, making it easier to produce such wind accretion systems. The lack of non-interacting binaries implied that fewer than $9.1 \%$ (90\% confidence) of SN lead to such systems with a companion stellar mass $>3 M_{\odot}$.

Identifying unbound binaries or higher multiplicity systems in older SNRs is more challenging because the star and the stellar remnant are no longer co-located. van den Bergh (1980) appears to have carried out the first search, looking for an excess of $\mathrm{O}$ and $\mathrm{B}$ stars in 17 SNRs and finding none. Guseinov et al. (2005) examine 48 SNRs for O and B stars based on their magnitudes, colors and proper motions, producing a list of candidates. Dincel et al. (2015) identified the most promising candidate to date, HD 37424 in G180.0-0.17 by back-propagating the proper motions of the star and the pulsar to find that they intersect near the center of the SNR. Boubert et al. (2017) examined 10 SNRs using the Tycho-Gaia astrometric solution (TGAS, Michalik et al. 2015) proper motions to search for stars which passed close to the centers of the SNRs on reasonable time scales. They identify four candidates, one of which is HD 37424. Lux et al. (2021) did the same for 12 SNRs using Gaia DR2 proper motions. Fraser \& Boubert (2019) used Gaia DR2 proper motions for both the stars and the compact objects in the Crab, Cas A and Vela SNRs, confirming the Kochanek (2018) result for the Crab and Cas A and only finding very low luminosity candidates in Vela.

Because many SNRs are asymmetric and distorted, using an estimate of the center of the SNR as an estimate of the explosion site is likely fraught for many SNRs unless a generous minimum distance is allowed to compensate for potential systematic problems. It is much safer to use the cases where a proper motion has been measured for the neutron star and we can simply search for intersections of the paths of the star and neutron star at some reasonable time in the past and distance from the center. Here we carry out this search for 7 neutron stars and 3 interacting binaries associated with SNRs that have sufficiently good proper motion measurements to carry out a search. We describe the sample and our methods in $\S 2$. In particular, we describe methods to evaluate the probability of false positives both as a general assessment for each SNR and for particular stellar candidates. In $\S 3$ we present the results for unbound binaries. In $\S 4$ we extend the search to fully unbound triples. In $\S 5$ we consider the one candidate in more detail. We discuss the results in $\S 6$.

\section{SEARCHING FOR UNBOUND SYSTEMS}

The 10 SNRs we examine are summarized in Appendix A, and their properties are given in Tables 1 and 2 Only two of the single neutron stars have directly measured parallaxes. For the remaining five we use rough distance estimates for the SNR expressed as parallaxes. For these cases the parallax entry in Table 2 is enclosed by parentheses. The three interacting binaries have Gaia proper motions and parallaxes.

We select nearby stars from Gaia EDR3 Gaia Collaboration et al. 2016, Gaia Collaboration et al. 2021), requiring them to have proper motions, all three Gaia magnitudes $\left(G, B_{P}\right.$ and $\left.R_{P}\right)$ and $G<18 \mathrm{mag}$. We include no restrictions on the RUWE statistic for the quality of the parallax, and for the present effort we can ignore the small systematic uncertainties in the parallax zero point and the statistical differences between the inverse parallax and the distance. We are assuming that any unbound star has moved away from the compact object, which is a very safe assumption since even a $10 \mathrm{~km} / \mathrm{s}$ velocity difference produces an angular separation of 2 arcsec at $10 \mathrm{kpc}$ after $10^{4}$ years.

The bright $G \simeq 3$ magnitude limit of Gaia could be a problem for nearby SNRs because it may lead to the exclusion of the most luminous stars. We addressed this by also searching the Hipparcos (Perryman et al. 1997) and Bright Star (Hoffleit \& Warren 1995) catalogs. For Hipparcos, we used the updated astrometric solution from van Leeuwen 
Table 1. Remnants

\begin{tabular}{ccr}
\hline SNR & Center & Radius (') \\
\hline G039.7-02.0 & $12: 12: 20+04: 55: 00$ & 42.4 \\
G069.0+02.7 & $19: 54: 50+33: 00: 30$ & 40.0 \\
G109.1-01.0 & $23: 01: 39+58: 53: 00$ & 16.5 \\
G130.7+03.1 & $02: 05: 34+64: 49: 50$ & 4.2 \\
G180.0-01.7 & $05: 40: 01+27: 48: 09$ & 100.0 \\
G205.5+00.5 & $06: 39: 00+06: 30: 00$ & 110.0 \\
G260.4-03.4 & $08: 22: 28-42: 57: 29$ & 30.0 \\
G263.9-03.3 & $08: 35: 21-45: 10: 35$ & 249.0 \\
G284.3-01.8 & $10: 18: 15-59: 00: 00$ & 12.0 \\
G296.5+10.0 & $12: 09: 40-52: 25: 00$ & 38.0 \\
\hline
\end{tabular}

Table 2. Compact Object Proper Motions and Parallaxes

\begin{tabular}{|c|c|c|c|c|c|}
\hline SNR & $\begin{array}{c}\text { Position } \\
\text { (J2000) }\end{array}$ & $\begin{array}{c}\text { PM RA } \\
\text { (mas/year) }\end{array}$ & $\begin{array}{c}\text { PM Dec) } \\
\text { (mas/year) }\end{array}$ & $\begin{array}{c}\varpi \\
(\mathrm{mas})\end{array}$ & Type \\
\hline G039.7-02.0 & $19: 11: 49.565+04: 58: 57.82$ & $-3.027 \pm 0.024$ & $-4.777 \pm 0.024$ & $0.118 \pm 0.023$ & binary, SS 433 \\
\hline G109.1-01.0 & $23: 01: 08.295+58: 52: 44.45$ & $-6.4 \pm 0.6$ & $-2.3 \pm 0.6$ & $(0.28 \pm 0.05$ & single \\
\hline G130.7+03.1 & $02: 05: 37.922+64: 49: 41.33$ & $-1.40 \pm 0.160$ & $0.540 \pm 0.575$ & $(0.36 \pm 0.14)$ & single \\
\hline G180.0-01.7 & $05: 38: 25.057+28: 17: 09.16$ & $-23.559 \pm 0.085$ & $52.766 \pm 0.079$ & $0.72 \pm 0.12$ & single \\
\hline G205.5+00.5 & $06: 32: 59.257+05: 48: 01.16$ & $-0.026 \pm 0.020$ & $-0.428 \pm 0.016$ & $0.540 \pm 0.023$ & binary, MWC 148 \\
\hline G284.3-01.8 & $10: 18: 55.587-58: 56: 45.98$ & $-6.454 \pm 0.013$ & $2.256 \pm 0.010$ & $0.227 \pm 0.010$ & binary, 2MASS J10185560-5856459 \\
\hline G296.5+10.0 & $12: 10: 00.913-52: 26: 28.30$ & $-12 \pm 5$ & $9 \pm 8$ & $(0.50 \pm 0.25)$ & single \\
\hline
\end{tabular}

Parallaxes in parentheses are based on rough distance estimates not direct measurements.

(2007). For the search regions used here, no bright stars were missing from the Gaia catalog.

We used PARSEC (Pastorelli et al. 2020) isochrones to group the stars into mass ranges based on their locations in an extinction-corrected $M_{G}$ versus $C=B_{P}-R_{P}$ color magnitude diagram. This is the only part of the calculation which uses distances, and simply using the inverse parallax here is sufficiently accurate because of the steepness of stellar mass-luminosity relations. We grouped them using limits of $1,2,3,4$ and $5 M_{\odot}$, with the various color-dependent absolute magnitude limits given in Table 3 As we noted in Kochanek et al. (2019), the lower mass stars will not yet be on the main sequence when the massive stars begin to explode as supernovae. These pre-main sequence stars lie almost a full magnitude redwards in color from the zero age main sequence. The jump downwards in the magnitude limit near $C=B_{P}-R_{P}=1$ is to ensure the inclusion of these pre-main sequence stars. For higher mass stars, the "dip" to fainter, redder pre-main sequence stars is no longer necessary. For the $>1 M_{\odot}$ case, we also exclude stars with $-1-3(C-1)<M_{G}<3+5(C-1)$ for $1.1<C<1.5$ to cut out the lower part of the red giant branch. The $N_{*}$ column of Table 4 gives the number of stars above each $M_{\text {lim }}$ that could have intersected the path of the neutron star in the last $10^{5}$ years at a velocity below $400 \mathrm{~km} / \mathrm{s}$ (see below). Magnitude limits are becoming important when the number of stars stops rapidly increasing with $M_{l i m}$.

For extinction estimates we used the three dimensional combined19 mwdust models (Bovv et al. 2016), which combine the Drimmel et al. (2003), Marshall et al. (2006) and
Green et al. (2019) models to provide extinction estimates for any sky position as a function of distance. We extracted the $\mathrm{V}$ band extinction, and use the PARSEC estimates of $A_{\lambda} / A_{V}$ for the Gaia EDR3 bands and an $R_{V}=3.1$ extinction law to convert the $\mathrm{V}$ band extinction to those for the $G, B_{P}$ and $R_{P}$ bands.

We first simply identify stars or pairs of stars whose parallaxes are consistent with the parallax of the neutron star. Given parallax estimates of $\varpi_{i} \pm \sigma_{\varpi, i}$ for $i=1,2$ (binary) or $i=1,2,3$ (triple) we adopt the joint best-fit parallax of

$$
\varpi=\left[\sum_{i} \frac{\varpi_{i}}{\sigma_{\varpi, i}^{2}}\right]\left[\sum_{i} \frac{1}{\sigma_{\varpi, i}^{2}}\right]^{-1}
$$

and the goodness of fit for this best-fit parallax,

$$
\chi_{\varpi}^{2}=\sum_{i}\left(\frac{\varpi_{i}-\varpi}{\sigma_{\varpi, i}}\right)^{2} .
$$

We initially keep all stars which as a potential binary companion satisfy $\chi_{\varpi}^{2}<9$. For the neutron stars without direct parallaxes, we use the weak constraints based on estimates of the distance to the SN discussed in the Appendix.

Next we restricted the sample to stars which could intersect the path of the neutron star in the time interval $-t_{m}<t<0$ at a stellar velocity less than $v_{*}<v_{\max }$. We again use a generous set of criteria because we are not yet using the actual stellar proper motions and their uncertainties, but this step does significantly reduce the numbers of stars which must be considered. We set $t_{m}=10^{5}$ years and $v_{\max }=400 \mathrm{~km} / \mathrm{s}$. If for distance $d$ the position difference between the neutron star and the star is $\overrightarrow{\Delta x}=d \overrightarrow{\Delta \alpha}$ 
Table 3. CMD Selection Limits

\begin{tabular}{ccrcccccc}
\hline Mass & Color & Mag & Color $)$ & Mag & Color & Mag & Color & Mag \\
\hline$>1 M_{\odot}$ & $<1.5$ & 5.5 & & & $1.5-2.0$ & $-1-3\left(B_{P}-R_{P}-1\right)$ & $>2$ & -4 \\
$>2 M_{\odot}$ & $<0.7$ & 2.0 & $0.7-1.1$ & 3.0 & $1.1-2.0$ & $-1-3\left(B_{P}-R_{P}-1\right)$ & $>2$ & -4 \\
$>3 M_{\odot}$ & $<0.7$ & 1.0 & $0.7-1.1$ & 2.0 & $1.1-2.0$ & $-1-3\left(B_{P}-R_{P}-1\right)$ & $>2$ & -4 \\
$>4 M_{\odot}$ & $<0.0$ & 0.5 & $0.0-1.3$ & -1.9 & $1.3-2.0$ & $-1-3\left(B_{P}-R_{P}-1\right)$ & $>2$ & -4 \\
$>5 M_{\odot}$ & $<0.0$ & -0.4 & $0.0-1.3$ & -1.9 & $1.3-2.0$ & $-1-3\left(B_{P}-R_{P}-1\right)$ & $>2$ & -4 \\
\hline
\end{tabular}

Mag gives the upper limit on $M_{G}$ for each $B_{P}-R_{p}$ color range.

where $\overrightarrow{\Delta \alpha}=\vec{\alpha}_{n s}-\vec{\alpha}_{*}$, and the velocity of the neutron star is $\vec{v}_{n s}=d \vec{\mu}_{n s}$, then the stellar velocity $v_{*}$ required to intercept the compact object at time $t$ is

$$
v_{*}^{2}(t)=v_{n s}^{2}+2 t^{-1} \vec{v}_{n s} \cdot \overrightarrow{\Delta x}+\Delta x^{2} t^{-2}
$$

which has a minimum of

$$
v_{* \text { min }}^{2}=\frac{\left|\overrightarrow{\Delta x} \times \vec{v}_{n s}\right|^{2}}{\Delta x^{2}}
$$

at time

$$
t_{* \min }^{-1}=-\frac{\overrightarrow{\Delta x} \cdot \vec{v}_{n s}}{\Delta x^{2}} .
$$

If the time of minimum velocity does not lie in the interval $-t_{m}<t_{* \min }<0$, then the minimum velocity for the star to intercept the compact object is just $v_{*}\left(-t_{m}\right)$, while if it does lie in the interval, the minimum velocity needed is $v_{* \min }$. The angular coordinate differences include the necessary $\cos \delta$ term for the separation in RA, and the positions of the stars passing the criteria are close enough to the neutron star that we need not do a full spherical trigonometry calculation.

We do not want to restrict our search to stars which can exactly intersect the neutron star at a particular time. If we allow binary separations of $a_{\max }$, then a star need only pass within the angular distance

$$
\sigma_{a}=10^{\prime \prime} 0\left(\frac{a_{\max }}{10^{4} \mathrm{AU}}\right)\left(\frac{\varpi}{\operatorname{mas}}\right)
$$

to potentially have been a binary companion of the neutron star. So we need a method that accounts for both the uncertainties in the proper motions and a finite angular separation at the time of explosion. We can account for both by introducing the true proper motions of the objects as an intermediate variable. Consider the motion in RA. Let the position separation be $\Delta \alpha_{12}=\alpha_{1}-\alpha_{2}$ (the $\cos \delta$ is again implicit), the measured proper motions in RA be $\mu_{\alpha, i}$, and the true proper motions in RA be $\mu_{\alpha, i}^{T}$. The true proper motion is constrained by the measured proper motions, and the separation as a function of time then depends on $\Delta \alpha_{12}-t\left(\mu_{\alpha, 1}^{T}-\mu_{\alpha, 2}^{T}\right)$. This leads to the fit statistic

$$
\begin{gathered}
\chi_{\alpha, 12}^{2}=\frac{\left(\mu_{\alpha, 1}-\mu_{\alpha, 1}^{T}\right)^{2}}{\sigma_{\alpha, 1}^{2}}+\frac{\left(\mu_{\alpha, 2}-\mu_{\alpha, 2}^{T}\right)^{2}}{\sigma_{\alpha, 2}^{2}} \\
+\frac{\left(\Delta \alpha_{12}-t\left(\mu_{\alpha, 1}^{T}-\mu_{\alpha, 2}^{T}\right)\right)^{2}}{\sigma_{a}^{2}}
\end{gathered}
$$

where the first two terms constrain the true proper motions using the measured proper motions and the last term constrains the closest approach given the true proper motions. If we optimize the statistic with respect to the unknown true proper motions,

$$
\chi_{\alpha, 12}^{2}=\frac{\left(\Delta \alpha_{12} / t-\Delta \mu_{\alpha, 12}\right)^{2}}{\sigma_{\alpha, 12}^{2}}
$$

where $\Delta \mu_{\alpha, 12}=\mu_{\alpha, 1}-\mu_{\alpha, 2}$, and

$$
\sigma_{\alpha, 12}^{2}=\sigma_{\alpha, 1}^{2}+\sigma_{\alpha, 2}^{2}+\sigma_{a}^{2} / t^{2} .
$$

If we now define $\Delta_{\alpha, 12}=\Delta \alpha_{12} / t-\Delta \mu_{\alpha, 12}$, then the overall statistic is

$$
\chi_{\mu}^{2}=\frac{\Delta_{\alpha, 12}^{2}}{\sigma_{\alpha, 12}^{2}}+\frac{\Delta_{\delta, 12}^{2}}{\sigma_{\delta, 12}^{2}},
$$

after including the analogous term in Declination. In essence, a $\chi_{\mu}^{2} \simeq 3$ means that the star can pass within distance $a_{\max }$ of the neutron star using proper motions that are each within $1 \sigma$ of the measured values. While the optimization of $\chi_{\mu}^{2}$ is formally non-linear in $t^{-1}$ due to Eqn. 9] the problem is easily solved iteratively: fix the time in Eqn.9 to determine a new optimal time, use this time to update Eqn. 9 and repeat. The ratio $t \sigma_{\mu} / \sigma_{a}$ of the two terms in Eqn. 9 indicates whether the position uncertainty due to the proper motions at closest approach $\left(t_{m} \sigma_{\mu}\right)$ is larger $\left(t_{m} \sigma_{\mu} / \sigma_{a}>1\right)$ or smaller $\left(t_{m} \sigma_{\mu} / \sigma_{a}<1\right)$ than the desired maximum separation $\sigma_{a}$. While we do not include the position uncertainties because they are unimportant, they can be included as an error term like $\sigma_{a}$.

With the uncertainties fixed, the closest approach time is

$$
t_{m}=-\frac{\Delta \alpha_{12}^{2} \sigma_{\delta, 12}^{2}+\Delta \delta_{12}^{2} \sigma_{\alpha, 12}^{2}}{\Delta \alpha_{12} \Delta \mu_{\alpha, 12} \sigma_{\delta, 12}^{2}+\Delta \delta_{12} \Delta \mu_{\delta, 12} \sigma_{\alpha, 12}^{2}}
$$

with a goodness of fit

$$
\chi_{12}^{2}=\frac{\left(\Delta \delta_{12} \Delta \mu_{\alpha, 12}-\Delta \alpha_{12} \Delta \mu_{\delta, 12}\right)^{2}}{\Delta \alpha_{12}^{2} \sigma_{\delta, 12}^{2}+\Delta \delta_{12}^{2} \sigma_{\alpha, 12}^{2}} .
$$

For equal uncertainties, the goodness of fit is basically just the amplitude of the proper motions perpendicular to the separation vector relative to the proper motion uncertainties, squared.

We use the same procedure to search for triples. The overall fit statistic replacing the first term of Eqn. 10 is

$$
\chi_{\alpha}^{2}=\frac{\sigma_{\alpha, 3}^{2} \Delta_{\alpha, 12}^{2}+\sigma_{\alpha, 2}^{2} \Delta_{\alpha, 13}^{2}+\sigma_{\alpha, 1}^{2} \Delta_{\alpha, 23}^{2}}{\sigma_{\alpha, 1}^{2} \sigma_{\alpha, 2}^{2}+\sigma_{\alpha, 1}^{2} \sigma_{\alpha, 3}^{2}+\sigma_{\alpha, 2}^{2} \sigma_{\alpha, 3}^{2}}
$$

with an equivalent term for the Declination. The definitions of the errors $\sigma_{\alpha, i}$ depend on how we want to restrict the separations. If we want to only restrict the minimum distance of the stars from the neutron star, then the uncertainties are $\sigma_{\alpha, 1}$ for the neutron star and $\sigma_{\alpha, i}^{2} \rightarrow \sigma_{\alpha, i}^{2}+\sigma_{a}^{2} / t^{2}$ for each of the two stars. Eqn. 13 then becomes identical to Eqn. 10 
Table 4. Searching For Disrupted Binaries

\begin{tabular}{|c|c|c|c|c|c|c|c|c|c|c|c|}
\hline \multirow[t]{2}{*}{ SNR } & \multirow{2}{*}{$\begin{array}{l}M_{l i m} \\
\left(M_{\odot}\right)\end{array}$} & \multirow[t]{2}{*}{$N_{*}$} & \multicolumn{3}{|c|}{$90 \%$} & \multicolumn{3}{|c|}{$95 \%$} & \multicolumn{3}{|c|}{$99 \%$} \\
\hline & & & $N_{o b s}$ & $N_{\text {ran }}$ & Prob. & $N_{o b s}$ & $N_{\text {ran }}$ & Prob. & $N_{o b s}$ & $N_{\text {ran }}$ & Prob. \\
\hline \multirow{5}{*}{ G039.7-02.0 } & $>5$ & 953 & 0 & 0.0 & - & 0 & 0.0 & - & 0 & 0.0 & - \\
\hline & $>4$ & 957 & 0 & 0.0 & - & 0 & 0.0 & - & 0 & 0.0 & - \\
\hline & $>3$ & 2202 & 0 & 0.0 & - & 0 & 0.0 & - & 0 & 0.0 & - \\
\hline & $>2$ & 2527 & 0 & 0.0 & - & 0 & 0.0 & - & 0 & 0.0 & - \\
\hline & $>1$ & 2641 & 0 & 0.0 & - & 0 & 0.0 & - & 0 & 0.0 & - \\
\hline \multirow[t]{5}{*}{ G069.0+02.7 } & $>5$ & 1382 & 0 & 0.1 & - & 0 & 0.2 & - & 2 & 0.7 & $16 \%$ \\
\hline & $>4$ & 1421 & 0 & 0.1 & - & 0 & 0.3 & - & 2 & 0.9 & $23 \%$ \\
\hline & $>3$ & 5178 & 2 & 1.9 & $55 \%$ & 3 & 3.2 & $61 \%$ & 20 & 14.9 & $12 \%$ \\
\hline & $>2$ & 9158 & 9 & 7.8 & $38 \%$ & 19 & 15.1 & $19 \%$ & 56 & 46.6 & $10 \%$ \\
\hline & $>1$ & 23748 & 66 & 83.7 & $98 \%$ & 111 & 128.6 & $95 \%$ & 213 & 227.5 & $84 \%$ \\
\hline \multirow[t]{5}{*}{ G109.1-01.0 } & $>5$ & 26 & 0 & 0.1 & - & 0 & 0.1 & - & 0 & 0.2 & - \\
\hline & $>4$ & 33 & 0 & 0.2 & - & 0 & 0.2 & - & 0 & 0.4 & - \\
\hline & $>3$ & 243 & 1 & 1.2 & $71 \%$ & 2 & 1.8 & $52 \%$ & 3 & 2.8 & $53 \%$ \\
\hline & $>2$ & 519 & 1 & 1.2 & $71 \%$ & 2 & 1.8 & $52 \%$ & 3 & 2.8 & $53 \%$ \\
\hline & $>1$ & 1741 & 1 & 1.2 & $71 \%$ & 2 & 1.8 & $52 \%$ & 3 & 2.8 & $53 \%$ \\
\hline \multirow[t]{5}{*}{ G130.7+03.1 } & $>5$ & 5 & 0 & 0.0 & - & 0 & 0.0 & - & 0 & 0.0 & - \\
\hline & $>4$ & 5 & 0 & 0.0 & - & 0 & 0.0 & - & 0 & 0.0 & - \\
\hline & $>3$ & 69 & 0 & 0.0 & - & 0 & 0.0 & - & 0 & 0.0 & - \\
\hline & $>2$ & 155 & 0 & 0.0 & - & 0 & 0.0 & - & 0 & 0.0 & - \\
\hline & $>1$ & 361 & 0 & 0.0 & - & 0 & 0.0 & - & 0 & 0.0 & - \\
\hline \multirow{5}{*}{ G180.0-01.7 } & $>5$ & 0 & 1 & 0.0 & $0 \%$ & 1 & 0.0 & $0 \%$ & 1 & 0.0 & $3 \%$ \\
\hline & $>4$ & 0 & 1 & 0.0 & $2 \%$ & 1 & 0.0 & $3 \%$ & 1 & 0.1 & $7 \%$ \\
\hline & $>3$ & 0 & 1 & 0.2 & $18 \%$ & 1 & 0.3 & $24 \%$ & 1 & 0.5 & $39 \%$ \\
\hline & $>2$ & 29 & 2 & 0.8 & $18 \%$ & 2 & 1.1 & $29 \%$ & 2 & 1.9 & $55 \%$ \\
\hline & $>1$ & 8973 & 8 & 9.7 & $75 \%$ & 9 & 13.6 & $92 \%$ & 18 & 20.9 & $77 \%$ \\
\hline \multirow[t]{5}{*}{ G205.5+00.5 } & $>5$ & 37 & 0 & 0.0 & - & 0 & 0.0 & - & 0 & 0.0 & - \\
\hline & $>4$ & 54 & 0 & 0.0 & - & 0 & 0.0 & - & 0 & 0.0 & - \\
\hline & $>3$ & 272 & 0 & 0.0 & - & 0 & 0.0 & - & 0 & 0.0 & - \\
\hline & $>2$ & 1067 & 0 & 0.0 & - & 0 & 0.0 & - & 0 & 0.0 & - \\
\hline & $>1$ & 11860 & 0 & 0.0 & - & 0 & 0.0 & - & 0 & 0.0 & - \\
\hline \multirow[t]{5}{*}{ G260.4-03.4 } & $>5$ & 147 & 0 & 0.2 & - & 0 & 0.3 & - & 0 & 0.7 & - \\
\hline & $>4$ & 168 & 0 & 0.6 & - & 0 & 0.7 & - & 0 & 1.2 & - \\
\hline & $>3$ & 658 & 0 & 1.9 & - & 1 & 2.8 & $94 \%$ & 8 & 5.6 & $20 \%$ \\
\hline & $>2$ & 1079 & 4 & 5.7 & $82 \%$ & 6 & 7.8 & $79 \%$ & 14 & 12.2 & $34 \%$ \\
\hline & $>1$ & 2679 & 28 & 31.8 & $77 \%$ & 35 & 39.4 & $78 \%$ & 54 & 54.4 & $54 \%$ \\
\hline \multirow[t]{5}{*}{ G263.9-03.3 } & $>5$ & 5 & 0 & 0.0 & - & 0 & 0.0 & - & 0 & 0.0 & - \\
\hline & $>4$ & 16 & 0 & 0.0 & - & 0 & 0.0 & - & 0 & 0.0 & - \\
\hline & $>3$ & 45 & 0 & 0.0 & - & 0 & 0.0 & - & 0 & 0.0 & - \\
\hline & $>2$ & 97 & 0 & 0.0 & - & 0 & 0.0 & - & 0 & 0.0 & - \\
\hline & $>1$ & 963 & 0 & 0.2 & - & 0 & 0.3 & - & 0 & 0.4 & - \\
\hline \multirow[t]{5}{*}{ G284.3-01.8 } & $>5$ & 322 & 0 & 0.0 & - & 0 & 0.0 & - & 0 & 0.0 & - \\
\hline & $>4$ & 539 & 0 & 0.0 & - & 0 & 0.0 & - & 0 & 0.0 & - \\
\hline & $>3$ & 1394 & 0 & 0.0 & - & 0 & 0.0 & - & 0 & 0.0 & - \\
\hline & $>2$ & 2016 & 0 & 0.0 & - & 0 & 0.0 & - & 0 & 0.0 & - \\
\hline & $>1$ & 2439 & 0 & 0.0 & - & 0 & 0.0 & - & 0 & 0.0 & - \\
\hline \multirow[t]{5}{*}{ G296.5+10.0 } & $>5$ & 63 & 0 & 0.0 & - & 0 & 0.0 & - & 0 & 0.0 & - \\
\hline & $>4$ & 63 & 0 & 0.0 & - & 0 & 0.0 & - & 0 & 0.0 & - \\
\hline & $>3$ & 606 & 0 & 0.2 & - & 0 & 0.3 & - & 0 & 0.3 & - \\
\hline & $>2$ & 1305 & 2 & 0.8 & $21 \%$ & 2 & 1.1 & $30 \%$ & 2 & 1.3 & $37 \%$ \\
\hline & $>1$ & 8372 & 15 & 13.9 & $42 \%$ & 18 & 15.8 & $33 \%$ & 22 & 17.5 & $17 \%$ \\
\hline
\end{tabular}


Table 5. Disrupted Binary Candidates

\begin{tabular}{ccccccccc}
\hline SNR & Gaia EDR3 ID & $\begin{array}{c}M_{\text {lim }} \\
\left(M_{\odot}\right)\end{array}$ & $\begin{array}{c}\text { False } \\
(\%)\end{array}$ & $\chi_{\varpi}^{2}$ & $\chi_{\mu}^{2}$ & $\begin{array}{c}t_{\min } \\
\left(10^{3} \mathrm{yr}\right)\end{array}$ & $R / R_{S N R}$ & $\begin{array}{c}\varpi \\
(\mathrm{mas})\end{array}$ \\
\hline G069.0+02.7 & 2034331673776151552 & 5 & 49 & 3.858 & 0.062 & -57.7 & 0.19 & $0.259 \pm 0.010$ \\
& 2034320300712461184 & 5 & 54 & 4.151 & 0.461 & -36.7 & 0.15 & $0.242 \pm 0.017$ \\
\hline G109.1-01.0 & 2013340789887658240 & 3 & 4 & 3.073 & 0.368 & -53.0 & 0.15 & $0.365 \pm 0.012$ \\
& 2013340381858998016 & 3 & 64 & 3.479 & 1.284 & -53.6 & 0.15 & $0.210 \pm 0.033$ \\
& 2013340175700592384 & 3 & 41 & 1.220 & 1.178 & -56.6 & 0.17 & $0.330 \pm 0.022$ \\
\hline G180.0-01.7 & 3441732292729818752 & 5 & 0 & 0.282 & 0.261 & -29.3 & 0.10 & $0.658 \pm 0.028$ \\
& 3441706518633166080 & 2 & 14 & 0.433 & 0.048 & -48.7 & 0.14 & $0.798 \pm 0.018$ \\
\hline G260.4-03.4 & 5526330988095223552 & 3 & 100 & 3.890 & 0.501 & -7.3 & 0.12 & $0.268 \pm 0.052$ \\
& 5526330472698907904 & 3 & 100 & 5.723 & 0.026 & -5.5 & 0.04 & $0.167 \pm 0.055$ \\
& 5526330403979671552 & 3 & 100 & 6.161 & 0.343 & -7.0 & 0.11 & $0.156 \pm 0.072$ \\
& 5526325387457616128 & 3 & 100 & 3.051 & 0.310 & -4.0 & 0.03 & $0.321 \pm 0.046$ \\
& 5526325284378390272 & 3 & 99 & 5.250 & 0.006 & -2.0 & 0.12 & $0.181 \pm 0.029$ \\
& 5526325284378390144 & 3 & 99 & 3.631 & 0.309 & -2.4 & 0.10 & $0.280 \pm 0.042$ \\
& 5526319099625741184 & 3 & 78 & 4.923 & 0.007 & -9.0 & 0.19 & $0.208 \pm 0.054$ \\
& 5526319095324073856 & 3 & 94 & 5.814 & 0.011 & -8.7 & 0.18 & $0.189 \pm 0.091$ \\
\hline G296.5+10.0 & 6125329220509401984 & 2 & 41 & 0.011 & 1.480 & -10.2 & 0.16 & $0.474 \pm 0.030$ \\
& 6125328945636431104 & 2 & 76 & 2.250 & 0.128 & -14.8 & 0.19 & $0.152 \pm 0.093$ \\
\hline \hline
\end{tabular}

Table 6. Disrupted Triple Candidates

\begin{tabular}{|c|c|c|c|c|c|c|c|c|c|}
\hline SNR & Gaia EDR3 ID & $\begin{array}{l}M_{l i m} \\
\left(M_{\odot}\right)\end{array}$ & $\begin{array}{c}\text { False } \\
(\%)\end{array}$ & $\chi_{\varpi}^{2}$ & $\chi_{\mu}^{2}$ & $\begin{array}{c}t_{\min } \\
\left(10^{3} \mathrm{yr}\right)\end{array}$ & $R / R_{S N R}$ & $\begin{array}{c}\varpi \\
(\mathrm{mas})\end{array}$ & Comment \\
\hline G069.0+02.7 & $\begin{array}{l}2034319682237156096,2034319510438472320 \\
2034331570696943232,2034330952221647232 \\
2034342943771429504,2034329848377469824 \\
2034342943771428992,2034331227099572480 \\
2034342943771428992,2034319299937887872\end{array}$ & $\begin{array}{l}3,3 \\
3,2 \\
3,1 \\
3,1 \\
3,1\end{array}$ & $\begin{array}{l}66,68 \\
65,30 \\
72,1 \\
56,65 \\
56,42\end{array}$ & $\begin{array}{l}5.689 \\
4.817 \\
3.994 \\
3.303 \\
1.804\end{array}$ & $\begin{array}{l}1.047 \\
3.804 \\
1.831 \\
3.225 \\
7.166\end{array}$ & $\begin{array}{l}-42.6 \\
-52.6 \\
-53.9 \\
-49.4 \\
-47.4\end{array}$ & $\begin{array}{l}0.10 \\
0.13 \\
0.15 \\
0.11 \\
0.10\end{array}$ & $\begin{array}{l}0.197 \pm 0.018 \\
0.271 \pm 0.021 \\
0.344 \pm 0.022 \\
0.447 \pm 0.012 \\
0.436 \pm 0.014\end{array}$ & \\
\hline G180.0-01.7 & 3441732292729818752,3442478238354719872 & 5,1 & 0,35 & 0.283 & 1.769 & -29.3 & 0.10 & $0.658 \pm 0.026$ & HD37424 \\
\hline G260.4-03.4 & 5526325353097874176,5526325250018655616 & 2,2 & 99,98 & 1.189 & 6.940 & -3.2 & 0.07 & $0.667 \pm 0.008$ & \\
\hline G296.5+10.0 & 6125329220509401984,6125328911271728768 & 2,1 & 41,92 & 2.128 & 4.753 & -10.0 & 0.16 & $0.496 \pm 0.026$ & \\
\hline
\end{tabular}

in the limit of making the proper motion uncertainties of one of the stars infinite. If you also add a term constraining the separation of the two stars by $\sigma_{a}$, then the uncertainties are $\sigma_{\alpha, i}^{2} \rightarrow \sigma_{\alpha, i}^{2}+\sigma_{a}^{2} / 3 t^{2}$ for all three objects. Here we use this latter form. The solution is again found by iteratively holding the uncertainties fixed, solving for $t_{m}$, and updating the uncertainties.

We consider the problem of false positives in two ways. First, we generally characterize each SNR by scrambling the stellar positions relative to their magnitudes, proper motions and parallaxes. We keep the latter three quantities tied because the uncertainties in the proper motions and parallaxes are in large part determined by the magnitude. While this is also true of the uncertainties in the positions, the position uncertainties are unimportant compared to the proper motion uncertainties and can be regarded as fixed. For each trial we assigned a random number to each star and assigned positions in the order of these random numbers. So if star \#1 appeared as the fiftieth entry in this list, it was assigned the position of star \#50. We rejected the occasional cases where a star would be assigned its true position, but we did not try to eliminate the occasional cases where multiple trials would consider the same random pairing. With $N_{*} \simeq 4000$ stars and $N_{t}=100$ trials, there are $N_{*} N_{t} \simeq 400,000$ random pairings of the neutron star with single stars of which we expect only $\sim 100$ duplicates. We do $N_{t}=100$ random trials for each SNR and count the number of candidates which have a position of closest approach inside $20 \%$ of the SNR radius and $\chi_{\varpi}^{2}+\chi_{\mu}^{2}$ smaller than several threshold values.

We can also evaluate the likelihood that a particular candidate is a false positive. This can be very different than the general probability of a false positive if a candidate has an unusual proper motion. We take all stars with $\chi_{\varpi}^{2}<9$ and within 1 mag of each candidate unbound binary star, and put it at the position of the candidate. We restrict the magnitude range so that the stars have similar kinematics and proper motion uncertainties. We then determine the fraction of these trials which would produce a $\chi_{\varpi}^{2}+\chi_{\mu}^{2}$ goodness of fit less than the larger of the fit statistic of the candidate or 2.706 (90\% confidence). We only do this for the unbound binary candidates because all candidate unbound triples are pairs of binary candidates. 


\section{BINARIES}

Table 4 summarizes the overall statistics of searching for unbound stars in each of the SNRs. For probability limits of $90 \%\left(\chi_{\varpi}^{2}+\chi_{\mu}^{2}<2.706\right), 95 \%$ (3.841) and 99\% (6.635), the table gives the number of stars considered, $N_{*}$, the number of actual candidates, $N_{\text {obs }}$, the average number of random candidates found per trial, $N_{\text {ran }}$, and the Poisson likelihood of finding $N_{o b s}$ or more candidates given an expectation value of $N_{\text {ran }}$, all as function of the minimum mass selection limit $M_{\text {lim }}$. The probability is meaningless for a bin with $N_{o b s}=0$, so no probability is given for these bins. These are all for $R / R_{S N R}<0.2, a_{\max }=10^{4} \mathrm{AU}$ and $t_{\min }>-10^{5}$ years. Note that there is little benefit from limiting the search to more compact binaries (smaller $a_{\max }$ ) because this scale does not matter once it is smaller than the position uncertainties created by back propagating the proper motions for tens of thousands of years.

The SNRs span a range of statistical properties from having essentially zero chance of a false positive for G263.9-03.3 because of its proximity and accurate neutron star parallax, to expecting large numbers of false positives for G069.0+02.7 because of the large number of stars and the absence of a neutron star parallax. The expected number of false positives rises as the mass limit is lowered because both the number of stars and the uncertainties in the Gaia parallaxes and proper motions increase, and it increases as the probability threshold is weakened. The numbers of observed candidates tracks the expected number from the random realizations extremely well, so the Poisson probabilities of finding at least as many candidates as observed are almost always significant. In fact, the only SNR with a candidate that is very unlikely to be a false positive is G180.0-01.7, and this is the Dincel et al. (2015) candidate HD 37424.

Since we are primarily interested in the more massive companions, we examined the candidates in the highest mass limit with candidates for each SNR, with the exception of G180.0-01.7, where use the mass limit producing a second candidate besides HD 37424. The properties of these candidates are given in Table 5. SNRs with no candidates are not included. In Table 5 we include the probability of obtaining a $\chi_{\varpi}^{2}+\chi_{\mu}^{2}$ value less than the larger of 2.706 and the actual value when assigning the same position the parallaxes and proper motions of some other star within $\pm 1 \mathrm{G}$ mag of the candidate. Essentially all the candidates except HD 37424 have positions that produce equally good or better fit statistics for significant fractions of randomly selected proper motions.

In the end, we reject all the candidates in Table 5 except HD 37424. The two candidates in G069.0+02.7 are likely false positives - their distances imply they are well behind the SNR, and the intercept times are somewhat young. For G109.1-01.0, one candidate does have a low false positive probability (4\%), but the parallaxes are all somewhat high or low and the intercept times are too old. Keep in mind that the false positive rates should be interpreted with a "trials" factor in mind - we are selecting multiple stars for having a path intersecting the neutron star from a still larger sample, so the probability of a $4 \%$ false positive probability star being a false positive is larger than $4 \%$ because of the multiple trials. The lower mass cut star we kept in G180.0-01.7 has a $14 \%$ false positive probability and an intercept time that is too old. G260.4-03.4 has a large number of lower mass candidates, all of which have very high false positive rates. Many of the parallaxes imply distances that are likely behind the SNR, although several have intercept times consistent with estimates for the age of the SNR. G296.5+10.0 has two candidates with high false positive probabilities but ages and parallaxes more or less consistent with estimates for the SNR. G130.7+03.1 and G263.9-03.3 have no candidates even for the $>1 M \odot$ mass cut.

Our various false positive tests make it clear that you will find candidates associated with any SNR if you search enough stars. The candidates in Table 5 come from going to lower and lower mass limits until there are candidates. They almost all have high false positive probabilities, so they are almost all likely to be false positives other than HD 37424 . However, with the available data and uncertainties, we cannot definitively prove this for particular stars. What we can conclude is that in this sample of ten SNRs, there is only one unbound companion with a mass $>5 M_{\odot}$, as we do think it is reasonable to exclude the two candidates associated with G069.0+02.7.

The unbound star search for the three SNRs with binaries is really a search for triple systems in which one star remains bound to the compact object and the other star becomes unbound. We find no such candidates for G039.7-02.0 (SS 433), G205.5+00.5 (HESS J0632+05 and MWC 148) or G284.3-01.8 (1FGL J1018.6-5856 and 2MASS J10185560-5856459). For SS 433, we carried out the search both for the Gaia EDR3 parallax and for the distances estimated from kinematic models of SS 433 by Blundell \& Bowler (2004) and Marshall et al. (2013). Table 4 given the results for the Gaia parallax distance. The limits for SS 433 should only be regarded as holding for $>5 M_{\odot}$ due to its larger distance and extinction compared to the other SNRs we consider. We can clearly reject the candidate HD 261393 found for G205.5+00.5 by Boubert et al. (2017). Its Gaia EDR3 parallax of $\varpi=0.923 \pm 0.025$ mas is inconsistent with that of MWC $148(\varpi=0.540 \pm 0.023$ mas $)$ at $\sim 130 \sigma$. The remaining Lux et al. (2021) candidates in this SNR also have parallaxes incompatible with MWC 148.

\section{DISRUPTED TRIPLES}

For the SNRs with single neutron stars, we can carry out the search for fully disrupted triples. We now keep candidates with $\chi_{\varpi}^{2}+\chi_{\mu}^{2}<9.210$ and allow pairings including all stars meeting the $>1 M_{\odot}$ mass cut. Because the two stars must have similar parallaxes and proper motions that intersect within $10^{5}$ years and $R / R_{S N R}<0.2$, the problem of false positives is greatly reduced over the search for unbound binaries. Nonetheless, it is still simply a question of how low a mass limit can be used before you have significant probabilities of false positives, not whether you will have false positives once you include enough stars.

The candidates are listed in Table 6. There are now only four SNRs with candidates despite the relaxed mass limits. Except for HD 37424, they all have high false positive probabilities, and G069.0+02.7 again has the most. Basically, since G069.0+02.7 has the largest number of stars which are unbound binary candidates, it is also much more likely that two of these candidates can have similar parallaxes and 
intercept times. Most of the candidates have rather high values for their $\chi_{\varpi}^{2}+\chi_{\mu}^{2}$ fit statistic.

Curiously, the best candidate again involves HD 37424, where there is a much lower mass star $\left(M_{\text {lim }}>1 M_{\odot}\right)$ with a consistent distance $\left(\chi_{\varpi}^{2}=0.3\right)$ and a good $\left(\chi_{\mu}^{2}=1.8\right)$, simultaneous intercept with the NS 29 thousand years ago close to the center of the remnant $\left(R / R_{S N R}=0.1\right)$. It does, however, have a significant false positive probability. Its kinematics would allow it to be in a wide binary with the neutron star progenitor and HD 37424. In the local standard of rest (see below in $\S 5$ ), its proper motion corresponds to a velocity of $\sim 22 \mathrm{~km} / \mathrm{s}$ as compared to $\sim 95 \mathrm{~km} / \mathrm{s}$ for HD 37424 . If we view these as circular velocities, and assume the neutron star progenitor and HD 37424 had similar masses, this would make the semi-major axis of the outer binary $\sim 40$ times that of the inner binary. Of course, the actual ratio would depend on the orbital phases at the time of explosion and the degree to which the system lay in the plane of the sky. There will, however, be a tendency for slower moving stars coincidentally close to the explosion site to produce false positives because the actual direction of their motions becomes relatively unimportant.

Like the examples of unbound binary candidates, we cannot prove that the unbound triple candidates in Table 6 are all false positives, although it seems likely. What we can say is that these seven SNRs contain no examples of disrupted massive star triple systems. Conservatively none of these neutron stars was in a triple containing two $>5 M_{\odot}$ stars in addition to the SN progenitor.

\section{$5 \quad$ HD 37424 AND PSR J0538+2817}

Fig. 1] shows a Gaia color-magnitude diagram (CMD) of all stars with parallaxes of $0.581<\varpi<0.758$ mas, which is nominally $\pm 200 \mathrm{pc}$ around the joint parallax $(\varpi=0.658 \pm$ 0.028 mas, or $1.52 \mathrm{kpc}$ ) of HD 37424 and the neutron star, angles $<3.77^{\circ}$ from the center of the SNR, which is $100 \mathrm{pc}$ at the nominal distance, and with $G<10 \mathrm{mag}$ (1 mag fainter than HD 37424). This yielded a sample of 50 stars. If we compare these stars to the PARSEC isochrones with $\log t=6.6,7.0,7.5,8.0$ and 8.5, we see that they are all consistent with $\log t \gtrsim 7.5$, and the initial mass of the most massive stars remaining on this isochrone is $9.1 M_{\odot}$. There are certainly no stars close to the $\log t=7.0$ isochrone where the initial mass of the most massive remaining star is $19.3 M_{\odot}$.

At the absolute magnitude of HD 37424, the $\log t \lesssim 7$ isochrones are nearly vertical, so any estimate of its age is largely determined by its color. Dincel et al. (2015) spectroscopically classified HD 37424 as a B0.5V star, and estimated a mass and luminosity of $M_{*} \simeq 13 M_{\odot}$ and $L_{*} \simeq$ $14000 L_{\odot}$ based on the calibrations of spectral types by Hohle et al. (2010).

We can construct a spectral energy distribution (SED) for HD 37424 spanning from $1570 \AA$ to $4.6 \mu \mathrm{m}$ using UV fluxes from Thompson et al. (1978), optical fluxes from NOMAD (Zacharias et al. 2005), near-IR fluxes from 2MASS (Skrutskie et al. 2006), and mid-IR fluxes from AllWise (Cutri et al. 2012). We used a temperature prior of $T_{*}=$ $26000 \pm 3000 \mathrm{~K}$ based on the spectral type, and an extinction prior of $E(B-V)=0.5 \pm 0.1$ based on the mwdust extinction estimate. The photometric errors were set to be the larger of the reported uncertainties and $10 \%$ with the exception of the $2740 \AA$ UV flux. The error for this band was set to $30 \%$ because it markedly disagrees with any smooth SED. The minimum photometric uncertainty is to compensate for systematic uncertainties. We fit the SED using Markov Chain Monte Carlo methods and Solar metallicity Castelli \& Kurucz (2003) atmosphere models to find that $T_{*}=27000 \pm 2600 \mathrm{~K}, L_{*}=16600 \pm 4500 L_{\odot}$, $R_{*}=5.9 \pm 0.3 R_{\odot}$ and $E(B-V)=0.34 \pm 0.04$. The goodness of fit is reasonable, with $\chi^{2}=9.7$ for 11 degrees of freedom (12 photometric measurements, 2 priors and 3 variables). The SED slightly constrains the temperature over the prior from the spectral type (B9). The SED requires less extinction than the mwdust estimate, which shifts the star redwards to colors more typical of the other nearby massive stars (see Fig. 1).

If we search for models with these luminosities and temperatures in the Solar metallicity PARSEC isochrones we can estimate the mass and age of the system. We selected models using a $\chi^{2}$ statistic to measure the distance of the model from the estimated stellar parameters and simply tracked the maximum and minimum values within a given $\chi^{2}$ limit. In matching to the isochrones we used the larger of the estimated uncertainties and $5 \%$ or $10 \%$ for the temperature and luminosity, respectively. For $\chi^{2}<1$, the current mass and age ranges were $11.1 M_{\odot}<M_{*}<13.9 M_{\odot}$ and $\log t<7.18$, while for $\chi^{2}<4$, the ranges were $10.0 M_{\odot}<M_{*}<15.3 M_{\odot}$ and $\log t<7.38$. There was no useful lower limit on the age. The upper age limits are certainly consistent with the star being the companion of a supernova, as the maximum initial masses for the $\log t=7.18$ and 7.38 isochrones are $14.0 M_{\odot}$ and $10.6 M_{\odot}$, respectively. These parameters are summarized in Table 7

We also modeled the SEDs of the 18 stars with mwdust extinction-corrected absolute magnitudes of $M_{G}<-2.5$ $\left(M_{G}<-3.0\right)$ and $B_{P}-R_{P}<1(>1)$. The limit on the blue main sequence is faint enough to include all stars which might eventually undergo core collapse, and the modest shift upwards in luminosity for redder colors was designed so that a few lower mass red giants were included to provide a check that they were lower mass without having to model many of them. This selection limit is shown in Fig. 1] For the red giants, we generally used APASS (Henden et al. 2016) instead of NOMAD for the optical magnitudes and the MARCS Gustafsson et al. 2008) atmosphere models. We had to broaden the photometric uncertainties of some bands to $20-30 \%$ to reach a reasonable $\chi^{2} / N_{\text {dof }}$. All the hotter stars had UV fluxes from Thompson et al. (1978), which provided enough temperature information to make it clear that the hottest stars were $\mathrm{B}$ and not $\mathrm{O}$ stars, consistent with the reported spectral types. This also allowed very robust individual extinction estimates. Table 7 reports the resulting temperatures and luminosities along with the $\chi^{2}<4$ range of PARSEC isochrone initial masses and ages to which they could correspond. The luminosities, temperatures, masses and ages of these stars, along with their spectral types and any interesting properties in the SIMBAD (Wenger et al. 2000) database, are also given in Table 7 and sorted by luminosity.

Most of the stars shift very little in the CMD with the directly estimated extinctions. The exception is TIC 3219130 where the mwdust extinction estimate of $E(B-$ 
Table 7. Luminous Stars Near HD 37424 and PSR J0538+2817

\begin{tabular}{lrccccl}
\hline \multicolumn{1}{c}{ Star } & $\chi^{2} / N_{\text {dof }}$ & $\log \left(T_{*} / \mathrm{K}\right)$ & $\left.\log \left(L_{*} / L_{\odot}\right)\right)$ & $M_{*} / M_{\odot}$ & $\log t$ & \multicolumn{1}{c}{ Comments } \\
\hline HD 39746 & 0.80 & $4.281 \pm 0.040$ & $4.761 \pm 0.124$ & $12.6-18.2$ & $6.99-7.22$ & B1II \\
V1164 Tau & 1.43 & $4.344 \pm 0.050$ & $4.524 \pm 0.151$ & $9.9-16.5$ & $7.00-7.39$ & A, Ell \\
HD 38658 & 0.68 & $4.179 \pm 0.033$ & $4.424 \pm 0.107$ & $9.9-13.6$ & $7.17-7.39$ & B3II \\
HD 36665 & 1.02 & $4.324 \pm 0.052$ & $4.379 \pm 0.163$ & $8.8-14.9$ & $7.05-7.49$ & B1, Be star \\
HD 38017A & 0.84 & $4.258 \pm 0.027$ & $4.289 \pm 0.066$ & $9.7-11.6$ & $7.28-7.42$ & B3V, visual binary \\
HD 37424 & 0.89 & $4.431 \pm 0.044$ & $4.219 \pm 0.128$ & $10.1-15.4$ & $<7.38$ & B9 \\
HD 35347 & 1.61 & $4.220 \pm 0.032$ & $3.837 \pm 0.081$ & $7.0-8.7$ & $7.49-7.69$ & B1V, Be star \\
ET Tau & 1.22 & $4.160 \pm 0.050$ & $3.819 \pm 0.160$ & $6.2-9.4$ & $7.44-7.81$ & B8, EB \\
HD 246821 & 0.84 & $3.886 \pm 0.043$ & $3.715 \pm 0.142$ & $5.8-8.9$ & $7.48-7.90$ & F0 \\
HD 248666 & 0.82 & $4.172 \pm 0.057$ & $3.708 \pm 0.167$ & $5.7-8.8$ & $7.50-7.89$ & B \\
HD 38749 & 0.75 & $3.897 \pm 0.023$ & $3.599 \pm 0.072$ & $6.1-7.4$ & $7.64-7.85$ & A5 \\
HD 247176 & 0.29 & $4.216 \pm 0.070$ & $3.536 \pm 0.204$ & $4.9-8.5$ & $6.69-8.04$ & B2V \\
HD 244610 & 1.46 & $4.076 \pm 0.073$ & $3.427 \pm 0.197$ & $4.6-7.5$ & $7.63-8.12$ & B1V, Be star \\
TIC 3219130 & 0.91 & $3.528 \pm 0.002$ & $3.337 \pm 0.014$ & $0.6-2.7$ & $8.84-9.99$ & M5 \\
HD 246370 & 0.17 & $3.831 \pm 0.022$ & $3.314 \pm 0.068$ & $5.3-6.3$ & $7.79-7.96$ & G5 \\
TIC 76026324 & 1.20 & $3.587 \pm 0.005$ & $3.295 \pm 0.013$ & $1.6-5.6$ & $7.91-9.37$ & M2/3 \\
HD 245247 & 0.81 & $3.816 \pm 0.012$ & $3.073 \pm 0.026$ & $4.7-5.3$ & $7.97-8.09$ & A7 \\
V399 Aur & 1.79 & $3.529 \pm 0.002$ & $2.856 \pm 0.016$ & $0.7-1.3$ & $9.66-9.99$ & M2/3, LPV \\
\hline
\end{tabular}

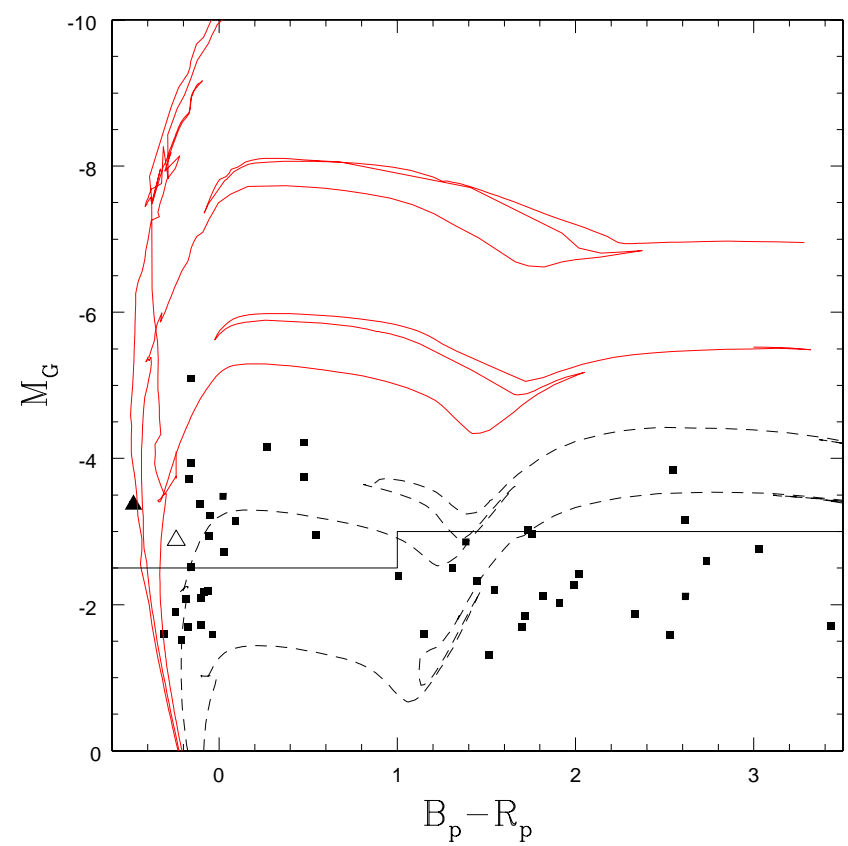

Figure 1. Extinction-corrected CMD of stars with $G<10$ mag within $\pm 200 \mathrm{pc}$ along the line-of-sight and $100 \mathrm{pc}$ in the plane of the sky of G180.0-01.7. The large filled (open) triangle is HD 37424 based the mwdust (SED fit) extinction estimate. The red solid PARSEC isochrones are for $\log t=6.6,7.0$ and 7.5, while the black dashed isochrones are for $\log t=8.0$ and 8.5. The maximum initial masses for stars remaining on the $\log t=7.0$, $7.5,8.0$ and 8.5 isochrones are $19.3,9.1,5.3$ and $3.4 M_{\odot}$, respectively. The SED models for the 18 stars above the horizontal lines are presented in Table 7

$V)=0.98$ is much too high - the SED implies $E(B-V)=$ 0.31 which makes the star a rather low mass red giant. The other two red giants (V339 Aur and TIC 76026324) are also relatively low mass, consistent with their locations on the CMD. The temperature estimates from the SEDs are gen-

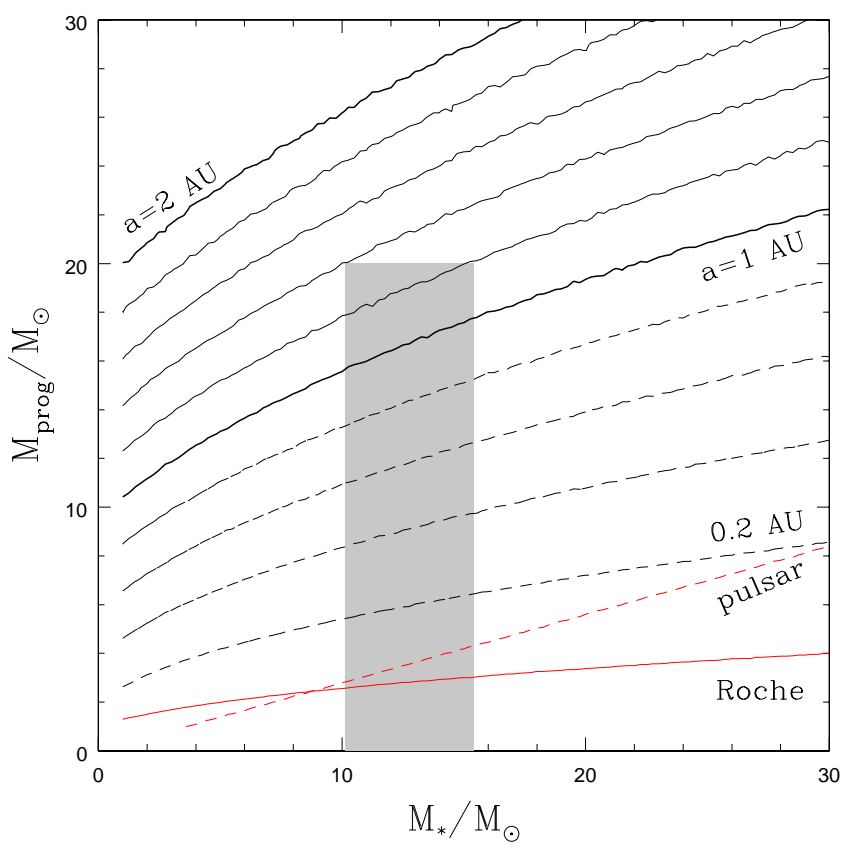

Figure 2. Semimajor axes as a function of the mass of HD 37424 $\left(M_{*}\right)$ and the mass of the SN progenitor at the time of explosion $\left(M_{p}\right)$. The shaded grey region is allowed by the fits to the SED of HD 37424 and the ages of nearby massive stars for the progenitor. Below the red Roche line, HD 37424 would fill its Roche lobe, and below the red dashed pulsar line the neutron star kick amplitude and direction become improbable. The kinematic uncertainties allow a $35 \%$ dispersion in the semimajor axis at fixed mass. There is a narrow band of acceptable pulsar solutions below the Roche line which has been excluded for clarity.

erally consistent with the spectroscopic classifications with the exception of V1164 Tau.

SIMBAD flags three stars as variables, V1164 Tau, ET Tau and V399 Aur. The classification of V1164 Tau as an ellipsoidal variable (ell) is from an automated classifier with 


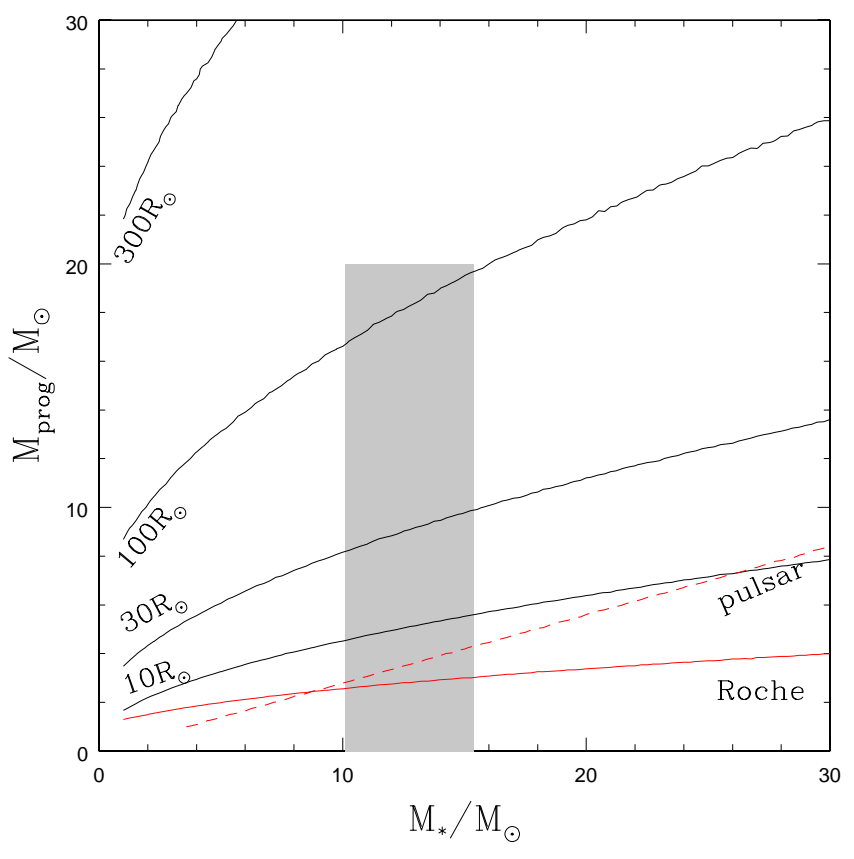

Figure 3. The Roche limit on the SN progenitor (black contours) over the same mass range as in Fig. 2 and with the same limits from the Roche radius of HD 37424 and the pulsar kinematics. The Roche limit on the progenitor is much smaller than the radii of red supergiants. The most likely scenario was that the progenitor was Roche lobe filling, transferring mass which "rejuvenated" HD 37424 to make it appear younger than other nearby massive stars and then leading to a Type IIL or IIb supernova. While not drawn, a $3 R_{\odot}$ Roche radius for the $\mathrm{SN}$ progenitor closely tracks the Roche radius limit for HD 37424.

no detailed analysis. As a luminous red giant, it is not surprising that V399 Aur is a long period variable. ET Tau is a fully analyzed eclipsing binary (EB), where Williamon et al. (2016) report parameters of $M_{1} \simeq 14.3, T_{1} \simeq 30300 \mathrm{~K}$, $L_{1}=30500 L_{\odot}$, and $M_{2}=6.3 M_{\odot}, T_{2}=15000 \mathrm{~K}$ and $L_{2}=6400 L_{\odot}$, respectively. This mass for the primary is much higher than found here $\left(5.8-8.9 M_{\odot}\right)$, but the SED also clearly rules out the high temperature and luminosity of the Williamon et al. (2016) model. In particular, the SED clearly breaks going into the UV rather than continuing the Rayleigh-Jeans rise that would be expected for $30000 \mathrm{~K}$ star.

Aside from HD 37424, all of the stars for which the age is constrained (i.e., excluding stars like HD 247176) favor ages $\log t \gtrsim 7.0$, and most are closer to $\log t=7.5$. There are clearly a number of stars which have masses $>10 M_{\odot}$, so it seems likely that the progenitor of PSR J0538+2817 had an initial mass between $\simeq 13 M_{\odot}$, since there are many remaining stars in this mass/age range, and $19 M_{\odot}$ since all the stars appear to be older than $\log t=7$. Dincel et al. (2015) argued for a mass range of $13 M_{\odot}$, their estimate of the mass of HD 37424 based on the spectral type, up to $25 M_{\odot}$ based on the absence of nearby O stars. Note that we are not using HD 37424 in setting this limit because we will next argue that the system must have been a mass transfer binary.

We would like to determine the properties of the pre-SN binary to constrain both the size of the orbit and the mass of the progenitor. Unfortunately, the geometry of the positions and proper motions can at best set a minimum projected size for the binary. The first problem is simply that parallaxes provide no useful information for constraining the line of sight separation at the time of the SNe. Then, in projection, it is only possible to rule out small separations, but not to prove them. A solution with an explosion at a projected separation of zero is allowed if there is a reasonable probability that the cross product of the present day separation and proper motion differences can be zero. This is true for HD 37424, although it need not be so. However, the raw probability of a given minimum projected separation $R_{\perp}$ is just proportional to $R_{\perp}$ because given a random choice for $R_{\perp}$ a value close to zero is unlikely. A Bayesian corrects for this by using logarithmic prior of $1 / R_{\perp}$, leading to a probability distribution which is independent of $R_{\perp}$ when it is small. For sufficiently large $R_{\perp}(\sim 30000 \mathrm{AU}$ for HD 37424), the distribution cuts off because large projected separations are unlikely. It is, after all, how the star was selected. However, while we select based on the minimum projected separation, the explosion need not happen at this closest approach distance, so a larger separation cannot be ruled out. In sum, the positions and proper motions would allow the progenitor system to be anything from a contact binary to a very, very wide binary.

It is really the velocity of the star which constrains the orbit, which is why Dincel et al. (2015) focus on the projected velocity of the star. They estimate a rest frame proper motion of $(10.0 \pm 0.8,-5.9 \pm 0.6)$ mas/year after correcting for Galactic rotation and Solar motion using a model. For the Gaia EDR3 parallax distance of $1.5 \mathrm{kpc}$, this corresponds to $87 \pm 9 \mathrm{~km} / \mathrm{s}$. They also measured an average heliocentric radial velocity of $-8.9 \pm 3.0 \mathrm{~km} / \mathrm{s}$. This implies an orbital separation smaller than the radius of a massive red supergiant, so Dincel et al. (2015) suggest that the progenitor was a naked helium star with a mass as low as $2 M_{\odot}$.

With Gaia, we can use the proper motions and radial velocities of nearby stars to define the local standard of rest. We selected stars in the same parallax range used for the luminous stars, a larger angular separation $\left(10^{\circ}\right)$ and apparent magnitudes within 1.5 mag of HD 37424 since kinematics depend on mass/luminosity. This yielded 523 stars with proper motions and 345 with radial velocities. HD 37424 does not have a Gaia radial velocity. These stars had a median proper motion of $(0.78,-3.3)$ mas/year with a dispersion (estimated from the central $68 \%$ of the sorted proper motions) of $(1.9,2.4) \mathrm{mas} /$ year, and a median radial velocity of $0.1 \mathrm{~km} / \mathrm{s}$ with a dispersion of $29.8 \mathrm{~km} / \mathrm{s}$. Note that the intrinsic dispersions are more important than the typical measurement uncertainties. Subtracting this from the proper motion of HD 37424 implies an SN local rest frame proper motion of $(11.5,-6.1) \mathrm{mas} /$ year with uncertainties set by the local proper motion dispersion, or $94 \pm 15 \mathrm{~km} / \mathrm{s}$. Similarly, subtracting the mean local RV from the Dincel et al. 2015) velocity for HD 37424 gives a local rest frame radial velocity of $-9.0 \pm 30.0 \mathrm{~km} / \mathrm{s}$. The larger dispersion in the radial velocity is puzzling.

The star has a small radial velocity compared to the velocities in the plane of the sky, and there are two arguments that the same is true of the present neutron star motions. First, Ng et al. (2007) argue that the pulsar wind nebula is seen almost edge on and aligned nearly perpendicular to the 
proper motion. Second, Yao et al. (2021) use scintillation of the pulsar signals off the near side of the SNR to estimate its distance from the neutron star and argue that the estimated distance puts the neutron star close to the center of the SNR and so implies that it has a low line-of-sight velocity. For the neutron star this could be entirely due to the kick, but it seems contrived to have the kick nearly cancel the motions along the line of sight. So for the present discussion, we assume that the pre-supernova binary effectively lay in the plane of the sky and that the orbit was circular.

Under these assumptions, the velocity of the star in the plane of the sky is

$$
\vec{v}_{*}=v_{o} \frac{M_{p}}{M_{T}}\left(\begin{array}{c}
\cos \theta \\
\sin \theta
\end{array}\right)
$$

and the velocity of the neutron star is

$$
\vec{v}_{N S}=-v_{o} \frac{M_{*}}{M_{T}}\left(\begin{array}{c}
\cos \theta \\
\sin \theta
\end{array}\right)+v_{k}\left(\begin{array}{c}
\cos \psi \\
\sin \psi
\end{array}\right)
$$

where $M_{*}$ is the mass of HD $37424, M_{p}$ is the mass of the SN progenitor at death, $M_{T}=M_{*}+M_{p}$ is the total mass, $v_{0}=\left(G M_{T} / a\right)^{1 / 2}$ where $a$ is the semimajor axis, $v_{k}$ is the neutron star kick velocity and $\theta$ and $\psi$ are the direction of the stellar velocity and the kick, respectively. As a function of the two masses, the proper motions and the estimated local standard of rest combine to give estimates of $a, v_{k}, \theta$ and $\psi$.

We have several additional constraints on these parameters. First, from the SED models we have an estimate of $M_{*}$. Second, the pulsar spin axis, generally believed to be perpendicular to the wind nebula, is nearly perpendicular to the proper motion of the pulsar, at a $\mathrm{PA}$ of $\theta_{\text {spin }}=-11^{\circ}$ or $12^{\circ}$ off the neutron star velocity vector ( $\mathrm{Ng}$ et al. 2007). This alignment is common and $\mathrm{Ng}$ \& Romani (2007) use the distribution of mis-alignments to try to constrain the origin of neutron star kicks. If we model the mis-alignments reported by Ng \& Romani (2007) with a Gaussian distribution corrected for the individual uncertainties, we find a best fit dispersion of $\sigma_{\psi}=17^{\circ}$. So we can constrain the angle $\psi$ as a position angle to be within $\sigma_{\psi}$ of $\theta_{\text {spin }}$. Third, there are constraints on the amplitudes of neutron star kick velocities, where we use the estimate that the distribution of 1D coordinate velocities can be modeled as a Gaussian with a dispersion of $\sigma_{k}=265 \mathrm{~km} / \mathrm{s}$ (Hobbs et al. 2005). Finally, we also have an estimate of the radius of HD 37424 from the SED models and can eliminate mass ranges where the Roche radius is smaller than the stellar radius, where we use the approximation for the mean Roche radius of Eggleton (1983).

Fig. 2]summarizes the results in the plane of the mass of HD $37424, M_{*}$, and the mass of the progenitor at death, $M_{p}$. A broader than plausible mass range is shown for both, with a gray shaded region giving the SED-based mass estimates for HD 37424 and a generous upper limit of $M_{p}<20 M_{\odot}$ based on the properties of the surrounding stars. Contours show the semi-major axis of the orbit implied by the masses and kinematics. For a given mass, the uncertainties in the kinematics produce a dispersion of $\simeq 35 \%$ around this central value across the plane of masses. For progenitor masses below the line labeled "Roche", HD 37424 would be overflowing its Roche lobe given its radius of $(5.9 \pm 0.3) R_{\odot}$. For progenitor masses below the line labeled "pulsar," the neu- tron star properties (kick and alignment) are unlikely. Over the rest of the plane, both the implied kick velocity and its alignment relative to the spin are consistent with expectations. There is a narrow band below the Roche curve where the pulsar kick velocity and direction are again consistent with expectations that we have suppressed for clarity.

In terms of the orbital kinematics, production of a neutron star in a supernova and the properties of the neutron star kick, there is a broad progenitor mass range leading to reasonable solutions. The problem, already noted by Dincel et al. (2015), is that a $10-20 M_{\odot}$ red supergiant should be significantly larger than these semimajor axes. Groh et al. (2013) find $547 R_{\odot}=2.5 \mathrm{AU}\left(931 R_{\odot}=4.3 \mathrm{AU}\right)$ for an initial mass of $10 M_{\odot}\left(20 M_{\odot}\right)$ and Sukhbold et al. (2016) have $414 R_{\odot}=1.9 \mathrm{AU}\left(1065 R_{\odot}=5.0 \mathrm{AU}\right)$. This problem cannot be solved by inclining the orbit out of the plane of the sky. Fig. 3illustrates this problem with contours for the Roche limit of the progenitor. Dincel et al. (2015) argue that the progenitor may have been a naked helium star at death, but this pushes into the very low pre-SN progenitor mass range where there are problems with the pulsar kinematics and the Roche limit of HD 37424.

The simplest solution is that the system was likely an interacting binary at death, with a Roche lobe-filling progenitor transferring mass to HD 37424. The mass transfer would help to explain why HD 37424 appears anomalously young and hot compared to the other stars in the field. The Roche limit is sufficiently small that the progenitor would have lost much of its hydrogen leading to a Type IIL or IIb supernova. In combination, these two classes represent $\sim 16 \%$ of ccSNe (Smith et al. 2011, Eldridge et al. 2013), so it is not a particularly improbable scenario for a randomly selected case.

\section{DISCUSSION}

We searched for unbound stellar companions to 7 non-binary neutron stars, two binary neutron stars and one binary black hole system that are both associated with SNRs and have proper motions. We identify only one convincing candidate, HD 37424 in G180.0-01.7, which was originally identified by Dincel et al. (2015) and also confirmed using different methods by Boubert et al. (2017). All the remaining candidates are lower mass and have high probabilities of being false positives. The mass limit to which companions can be ruled out depends on the SNR, but a conservative limit is that there are no unbound binary or triple systems other than HD 37424 with masses $\gtrsim 5 M_{\odot}$.

We estimate that HD 37424 has a mass of $10-15 M_{\odot}$ and that it was probably in a mass transfer binary with a semi-major axis of $\sim 1$ AU that would partly strip the neutron star progenitor and lead to a Type IIL or IIb SN. The progenitor was likely a $13-19 M_{\odot}$ star at birth based on the properties of the stars within $100 \mathrm{pc}$ of the explosion. These conclusions agree with Dincel et al. (2015) except for the nature of the progenitor at death, where Dincel et al. (2015) suggest it was a fully stripped helium star.

By combining this work, Kochanek (2018) and Kochanek et al. (2019), we can constrain all of the possibilities for the binary properties and outcomes of explosions producing neutron stars. In Kochanek (2018) we showed 
Table 8. Constraints on Binaries

\begin{tabular}{|c|c|c|c|c|}
\hline \multirow[t]{2}{*}{ Case } & \multicolumn{2}{|c|}{ Non-Interacting Incomplete } & \multicolumn{2}{|c|}{ Non-Interacting Complete } \\
\hline & Median & $90 \%$ Confidence & Median & $90 \%$ Confidence \\
\hline Not Binary at Death & $72.0 \%$ & $52.2 \%-86.4 \%$ & $76.0 \%$ & $56.0 \%-89.2 \%$ \\
\hline Bound Binary & $13.9 \%$ & $5.4 \%-27.2 \%$ & $9.5 \%$ & $3.6 \%-19.7 \%$ \\
\hline Interacting Binary & $10.2 \%$ & $3.2 \%-22.3 \%$ & $5.8 \%$ & $1.8 \%-13.1 \%$ \\
\hline Non-Interacting Binary & & $<8.5 \%$ & & $<8.9 \%$ \\
\hline Unbound Binary & $12.5 \%$ & $2.8 \%-31.3 \%$ & $13.2 \%$ & $3.0 \%-32.8 \%$ \\
\hline
\end{tabular}

that the Crab, Cas A and SN 1987A were not binaries at the time of explosion. Because these SNRs are so young, there is no need for proper motion information. We are assuming that SN 1987A produced a neutron star (see, e.g., Cigan et al. 2019, Greco et al. 2021). In Kochanek et al. (2019) we searched for surviving binaries starting from an initial sample of 49 SNRS. Of these, 23 contained compact objects where it was practical to carry out a search, and this included three previously identified HMXBs. If we drop SS 433 as a black hole system, then there were two interacting binaries and no non-interacting binaries in a sample of 22 explosions producing neutron stars.

We can combine all of these results to jointly estimate the fraction $f_{n}=1-f_{u}-f_{b}$ of SN producing neutron stars that are not binaries at the time of the SN, the fraction $f_{u}$ leading to binaries that are unbound after the explosion, the faction $f_{b}=f_{i}+f_{p}$ leading to binaries that are bound, where fraction $f_{i}$ produce interacting binaries and $f_{p}$ produce noninteracting (passive) binaries. The multinomial probability distribution is

$$
f_{n}^{9}\left(1-f_{b}\right)^{11} f_{u} f_{i}^{2}
$$

since we have 9 SNR containing neither bound nor unbound binaries, 11 which contain no bound binary but could be unbound binaries, 1 unbound binary and two interacting binaries. Again, we conservatively mean stellar companions $\gtrsim 5 M_{\odot}$, although the mass limits for individual systems can be significantly tighter. This case, "Non-Interacting Incomplete" in Table 8 assumes that the presence of the interacting companion does not bias whether the interacting binary was included in the sample of 23 SNRs where Kochanek et al. (2019) searched for bound companions. Using uniform priors for the fractions, the probability can be marginalized to obtain the probability distribution for each of these fractions, with the median values and $90 \%$ confidence ranges given in Table 8 .

If, on the other hand, interacting binaries are so easy to detect due to their X-ray, $\gamma$-ray or radio emission that the 23 SNRs analyzed by Kochanek et al. (2019) included all interacting binaries in the parent sample of 49 SNRs. If so, we have overestimated the probability of producing an interacting binary. If we assume all interacting binaries were included (case "Non-Interacting Complete") then there were 2 interacting neutron star binaries in a sample of 43 SNRs after making a rough correction for contamination by Type Ia SN (see Kochanek et al. 2019). In this case, the multinomial probability distribution becomes

$$
f_{n}^{9}\left(1-f_{b}\right)^{11}\left(1-f_{i}\right)^{20} f_{u} f_{i}^{2}
$$

because there are an additional 20 SNRs known not to contain interacting binaries. The results for this case are also given in Table 8 and the true answer should be bounded by these two limits.

We will not attempt a similarly complete sub-division into cases for the question of triple systems at death in part because we lack information on whether the interacting binaries and HD 37424 have distant bound companions. However, of the 10 systems where the compact object is not in a binary (the seven here plus the Crab, Cas A and SN 1987A), none are fully unbound triples. So of SN that do not leave bound binaries, less than $18.9 \%$ can be fully unbound massive star triples. Of the 3 systems which are interacting binaries, none is a partially unbound triple. Thus, of SN leaving interacting binaries, less than $<43.7 \%$ can have an unbound triple companion. Note that these are fractions of systems which are not binaries or binaries after the explosion, while the statistics for binaries in Table 8 are all as fractions of SN.

While there will be a point where systematic problems due to any correlation between the detectable life times of SNRs and any of the binary properties we consider will become important, statistical uncertainties are likely dominant at present (see the discussion in Kochanek et al. 2019). It certainly seems possible to significantly improve the statistics simply by identifying more compact objects in SNRs with reasonable distances and extinctions and then measuring their proper motions. In Kochanek et al. (2019) we found that the problem for many of the SNRs which could be studied was that there were multiple, faint, X-ray sources superposed on the remnant. Detecting a proper motion would clearly separate the neutron star from the contaminating background sources. Where the neutron star is already detected, a sufficient amount of time has likely passed since the original observations that proper motions can be measured or at least tightly restricted. Simply knowing that the neutron star has (say) moved less than $0^{\prime \prime} 1$ in 20 years restricts the allowed position $10^{4}$ years ago to under an arcminute.

The biggest cause of false positives in searching for unbound stars is the lack of good distances to the SNRs. In theory this can be addressed by searching for absorption features associated with the SNR in the spectra of superposed stars. This approach appears to have worked quite well for the Vela SNR, where the estimated distance of $250 \pm 30$ pc by Cha et al. (1999) based on the appearance of high velocity components in the $\mathrm{Na} \mathrm{D}$ and Ca II absorption lines of more distant stars agrees almost exactly with the Dodson et al. (2003) pulsar parallax distance $\left(\varpi^{-1}=286 \pm 16 \mathrm{pc}\right)$. The absorption lines of the background stars to Vela are also unusual because many of them are significantly time variable (see Kameswara Rao et al. 2020 most recently). With Gaia distances and fiber-fed echelle spectrographs this would ap- 
pear to be a straight forward approach to measuring the distances to many SNRs.

Improving the constraints on fully unbound triples is much easier than doing so for unbound binaries because accurate distances to the SNR and pulsar proper motions are less needed for the control of false positives. Particularly if restricted to relatively massive and rare stars (say $>5 M_{\odot}$ ), the requirement that two stars with Gaia proper motions and parallaxes have consistent distances and paths that intersect near the center of the SNR at some point in the last $10^{5}$ years will greatly suppress or eliminate false positives. The same is also true for a partly unbound binary where the compact object is in a binary because Gaia supplies the parallax and proper motion of the binary companion to the compact object.

HD 37424 is a very convincing case of an unbound binary companion, but what can be done both to strengthen the evidence and use it to learn something about binary evolution, supernovae and supernovae in binaries? Dincel et al. (2015) looked for high velocity absorption features in their echelle spectra of HD 37424 and found nothing but note that the signal-to-noise ratio of the spectrum was relatively low. Finding such features, would help to confirm the hypothesis and it would be trivial to obtain far lower noise spectra from the ground. It might be better, however, to look in the UV with HST where there are many more lines (see, e.g., the study of the SN 1006 SNR by Winkler et al. 2005). An unusually fast rotation rate would help to confirm the interacting binary scenario, but a low estimate of $v \sin i$ will be difficult to interpret given the evidence that the binary likely lay in the plane of the sky. The supernova shock wave affects the structure of the outer layers and the luminosity of the star, but any time evolution from the relaxation back to steady state is likely impossible to detect with the passage of 30,000 years (see, e.g., Ogata et al. 2021). The best route may be to look for abundance anomalies, as several HMXBs are known to have anomalous $\alpha$-element abundances presumably due to capturing some of the ejecta from the SN (e.g., Israelian et al. 1999, Orosz et al. 2001).

\section{ACKNOWLEDGMENTS}

CSK thanks K. Belczynski for help with BSP models when this project was headed in a somewhat different direction, and R. de Stefano, Jennifer Johnson, M. Moe and M. Pinsonneault for various discussions. CSK is supported by NSF grants AST-1814440 and AST-1907570. This work has made use of data from the European Space Agency (ESA) mission Gaia (https://www.cosmos.esa.int/gaia), processed by the Gaia Data Processing and Analysis Consortium (DPAC, https://www.cosmos.esa.int/web/gaia/dpac/consortium).

Funding for the DPAC has been provided by national institutions, in particular the institutions participating in the Gaia Multilateral Agreement. This research has made use of the SIMBAD database, operated at CDS, Strasbourg, France

\section{APPENDIX A: SAMPLE}

We examined 10 SNRs where there is a proper motion measurement either for the neutron star (7 systems) or its binary companion (3 systems) that also have distances and extinctions small enough to search for stellar companions using Gaia EDR3 parallaxes and proper motions. For statistical analyses we also include the Crab (G184.6-05.8), Cas A (G117-02.1) and SN 1987A. Because these SNRs are very young, the plausible regions in which any former companion could lie are so small that Kochanek (2018) (also see, Kerzendorf et al. 2019, Fraser \& Boubert 2019) could demonstrate that there were no bound or unbound former companions down to mass ratios $\lesssim 0.1$ even in the absence of proper motion data.

\section{A1 G039.7-02.0}

Contains the interacting compact object binary SS 433 (for a review, see Margon 1984). We us the position and size of the SNR from Green (2019). SS 433 is more distant $\left(\varpi^{-1}=8.5 \mathrm{kpc}\right)$ and highly extincted (mwdust $E(B-V) \sim 1$ to $2 \mathrm{mag}$ ) than the other SNRs we consider here, so we selected stars to $G<20$ mag instead of $G<18$ mag. Even so, this means we are really only sensitive to stars in the $M>5 M_{\odot}$ bin. To limit the number of stars given the faint magnitude limit, we only considered stars with $0<\varpi \leq 0.25$ mas, corresponding to the $\sim 5 \sigma$ range of the Gaia EDR3 parallax. The distance implied by the Gaia EDR3 parallax (6.1 to $13.9 \mathrm{kpc}$ at $2 \sigma$ ) significantly disagrees with distance estimates from kinematic models of the SS 433 jets $(5.5 \pm 0.2$, Blundell \& Bowler 2004, $4.5 \pm 0.2 \mathrm{kpc}$, Marshall et al. 2013) but the parallax $R U W E=1.24$ is not anomalously high. As a precaution, we also searched for unbound stars using a parallax of $0.208 \pm 0.042$ mas, which encompasses the kinematic distance estimates and again found no candidates. The age of the remnant is estimated to be anywhere from $10^{4}$ to $10^{5}$ years (see, e.g., Panferov 2017).

\section{A2 G069.0+02.7 (CTB 80)}

Contains the radio pulsar PSR B1951+32 (Kulkarni et al. 1988). which has a proper motion of $(-28.8 \pm 0.9,-14.7 \pm$ 0.9) mas/year (Zeiger et al. 2008). There is no parallax for the pulsar. Koo et al. (1993) roughly estimate the distance to CTB 80 to be $d \simeq 2 \mathrm{kpc}$, while Leahy \& Ranasinghe (2012) estimate a distance of $1.5_{-0.4}^{+0.6} \mathrm{kpc}$. Kulkarni et al. (1988) estimate $1.4 \mathrm{kpc}$ from the dispersion measure to the pulsar. We adopt a loose limit on the "parallax" of the SNR of $\varpi=0.75 \pm 0.25$ mas which encompasses 1 to $2 \mathrm{kpc}$ at $1 \sigma$. We adopt 19:54:50 33:00:30 for the center of the SNR from Zeiger et al. (2008), and a diameter of 80 arcmin from Green (2014). The pulsar has a spin-down age of $\tau_{c}=P / 2 \dot{P}=$ 107 thousand years (Fruchter et al. 1988). Koo et al. (1990) estimate an age from the size and expansion velocity of the SNR of 77 thousand years for a distance of $2 \mathrm{kpc}$. For a limiting magnitude of $G<18 \mathrm{mag}$, the sample of $\sim M_{\odot}$ stars will be incomplete, but the false positive rate is also too high to search for such low mass companions. 


\section{A3 G109.1-01.0}

This SNR contains the anomalous X-ray pulsar 1E 2259+586 (Gregorv \& Fahlman 1980) which has a proper motion of $(-6.4 \pm 0.6,-2.3 \pm 0.6)$ mas/year (Tendulkar et al. 2013). Verbiest et al. (2012) estimate a distance of $4.1 \pm 0.7 \mathrm{kpc}$ Kothes \& Foster (2012) and Sánchez-Cruces et al. (2018) argue for a lower distance of $3.2 \pm 0.2 \mathrm{kpc}$. We adopt a "parallax" of $\varpi=0.28 \pm 0.05$ which spans 3.2 to $4.3 \mathrm{kpc}$ at $1 \sigma$. Kothes et al. (2006) derive a center of 23:01:39 +58:53:00 with a diameter of 33 arcmin. The spin down age is 230 thousand years, but estimates based on the remnant are generally 10 to 20 thousand years (see, Sánchez-Cruces et al. 2018). $N(H)=(0.93 \pm 0.04) \times 10^{22} \mathrm{~cm}^{2}$ (Patel et al. 2001), corresponding to $E(B-V)=1.60 \pm 0.07$. For a limiting magnitude of $G<18 \mathrm{mag}$ and a distance of $4 \mathrm{kpc}$ we can only search for $>4 M_{\odot}$ companions.

\section{A4 G130.7+03.1 (3C58, SN 1181)}

This SNR contains the X-ray pulsar PSR J0205+6449 (Murrav et al. 2002), which has a proper motion of $(-1.40 \pm 0.16,0.54 \pm 0.58)$ mas/year Bietenholz et al. (2013). Roberts et al. (1993) estimate a kinematic (HI) distance of $3.2 \mathrm{kpc}$, while Camilo et al. (2002) obtain $4.5_{-1.2}^{+1.6} \mathrm{kpc}$ based on the dispersion measure to the pulsar, and Kothes \& Foster (2012) argues for $2 \mathrm{kpc}$. The age is also uncertain. The spin down age is 5.4 thousand years (Livingstone et al. 2009) while it would only be 0.84 thousand years old if associated with SN 1181 (Clark \& Stephenson 1977). Studies of the remnant (e.g., Bietenholz 2006) generally argue that the SNR must be several thousand years old. We adopt a "parallax" of $0.36 \pm$ 0.14 mas which spans 2.0 to $4.7 \mathrm{kpc}$ at $1 \sigma$. Gotthelf et al. (2007) propose 02:05:33.97 64:49:50 as the explosion center 1 and the asymmetric remnant has an average diameter of approximately 8.1 arcmin (Revnolds \& Aller 1988). Slane et al. (2004) find $N(H)=(4.5 \pm 0.1) \times 10^{21} \mathrm{~cm}^{2}$, corresponding to $E(B-V) \simeq 0.77 \pm 0.02$. For a limiting magnitude of $G<18 \mathrm{mag}$ and a distance of $5 \mathrm{kpc}$ we can only search for $>3 M_{\odot}$ companions.

\section{A5 G180.0-01.7}

This SNR contains the radio pulsar PSR J0538+2817 (Anderson et al. 1996). Ng et al. (2007) measured VLBI parallax and proper motions of $\varpi=0.68 \pm 0.15$ mas and $(-23.53 \pm 0.16,52.59 \pm 0.13)$ mas/year, and Chatteriee et al. (2009) measured $\varpi=0.72_{-0}^{+0.12}$ and $\left(-23.57 \pm 0.10,52.87_{-0.10}^{+0.09}\right) \mathrm{mas} /$ year. The Chatteriee et al. (2009) analysis appears to use the $\mathrm{Ng}$ et al. (2007) data but considers more sources of systematic error, so we use their values and the larger values for the asymmetric errors. Kramer et al. (2003) estimate that the center of the SNR is at 05:40:01 +27:48:09, and Dincel et al. (2015) estimate that the diameter is 200 arcmin. Based on the disrupted

1 The text of Gotthelf et al. (2007) gives the declination of the center as 63:49:50, but this appears to be a misprint given the figures and the discussion. binary, Dincel et al. (2015) estimate an age of (30 \pm 4$)$ thousand years. The pulsar has a spin down age of 620 thousand years (Lewandowski et al. 2004). Based on a Sedov model of the SNR, Sofue et al. (1980) estimated the age of the SNR as 200 thousand years for a distance of $1.6 \mathrm{kpc}$. $\mathrm{Ng}$ et al. (2007) also find $N(H) \simeq(2.7 \pm 0.3) \times 10^{21} \mathrm{~cm}^{2}$, implying $E(B-V) \simeq 0.5 \pm 0.1$. Even at a higher distance of $1 \mathrm{kpc}$ we can search for companions down to $>1 M_{\odot}$.

Dincel et al. (2015) and later Boubert et al. (2017) identify HD 37424 as a candidate disrupted binary companion. HD 37424 has a Gaia EDR3 parallax and proper motion of $\varpi=0.654 \pm 0.028 \mathrm{mas}$ and $(12.229 \pm 0.036$, $-9.407 \pm 0.017) \mathrm{mas} / \mathrm{year}$. The parallaxes are mutually consistent to $0.5 \sigma$. Dincel et al. (2015) were concerned that the stars HD 36665 and HD 37318 show absorption features due to the SNR but had distance estimates that placed them in the foreground. In Gaia EDR3, these stars have parallaxes of $\varpi=0.702 \pm 0.031$ mas and $\varpi=0.566 \pm 0.030$ mas, so statistically, HD 36665 could be more distant, and HD 37318 clearly is more distant than the SNR.

\section{A6 205.5 +00.5 (Monoceros Loop)}

The Monoceros loop is associated with the $\gamma$-ray source HESS J0632+057. The SNR was identified prior to the discovery of the $\gamma$-ray source. Hinton et al. (2009) also identified it as an X-ray source and suggested that it was in a binary with the massive star MWC 148. The high energy emissions are believed to be due to interactions between a pulsar and the stellar wind (see the review by Dubus 2013). We use the center and size of the SNR from Green (2019). Boubert et al. (2017) identify HD 261393 as a candidate disrupted binary companion in this SNR but its parallax is inconsistent with that of MWC 148 (see text). Welsh et al. (2001) estimate an age of 30 to 150 thousand years.

\section{A7 G260.4-03.4 (Puppis A)}

Puppis A contains the X-ray pulsar PSR J0821-4300 (Gotthelf \& Halpern 2009) where Maver \& Becker (2021) measure a proper motion of $\left(-74.2_{-7.7}^{+7.4}\right.$, $\left.-30.3_{-6.2}^{+6.2}\right)$ mas/year. We round the asymmetric uncertainties upwards Revnoso et al. (2017) estimate a kinematic distance to the SNR of $1.3 \pm 0.3 \mathrm{kpc}$, although earlier estimates in Reynoso et al. (2003) were higher, at 2.2 kpc. We adopt a "parallax" of $\varpi=0.75 \pm 025$ mas which spans 1 to $2 \mathrm{kpc}$ at $1 \sigma$. Winkler et al. (1988) found an explosion center of 08:22:27.5 - 42:57:29 based on the proper motions of optical filaments in the SNR and Green (2014) gives a diameter of 60 arcmin. The motions of the optical filaments also imply an age of $3700 \pm 300$ years (ignoring deceleration). Hui \& Becker (2006) find $N(H)=(3.7 \pm 0.1) \times 10^{21} \mathrm{~cm}^{2}$ corresponding to $E(B-V)=0.63 \pm 0.02$. For $G<18 \mathrm{mag}$ and a distance of $2 \mathrm{kpc}$, we can search for companions down to $>2 M_{\odot}$.

\section{A8 G263.9-03.3 (Vela)}

The Vela pulsar (PSR J0835-4510, Large et al. 1968) has a well-measured parallax $(\varpi=3.5 \pm 0.2)$ and proper motion $(-49.68 \pm 0.06,29.9 \pm 0.1) \mathrm{mas} /$ year from Dodson et al. 
(2003). We could find no explicit estimate for the center of the Vela SNR. Aschenbach et al. (1995) clearly made such an estimate, as they report it is $25 \pm 5$ arcmin from the pulsar, but do not include the actual coordinates. They report the diameter of the remnant as 8.3 degrees. The position given by Green (2014) is visibly offset from the center of the X-ray image of the SNR in Aschenbach et al. (1995) and the reported diameter is only 4.3 degrees. We set the center of the SNR to be 25 arcmin backwards from the neutron star along its proper motion vector, which should be the Aschenbach et al. (1995) center and use their larger diameter. Taylor et al. (1993) give a spin down age of 11 thousand years. Estimates based on the properties of the SNR by Aschenbach et al. (1995) range from 8 to 37 thousand years. $N(H)=(3.0 \pm 0.3) \times 10^{20} \mathrm{~cm}^{2}$ (Pavlov et al. 2001). This column density implies $E(B-V) \simeq 0.05$ mag. With its proximity and low extinction, there is no problem searching for companions down to $>1 M_{\odot}$.

\section{A9 G284.3-01.8}

This SNR is associated with the $\gamma$-ray source 1FGL J1018.6-5856, and it was identified as an HMXB by Corbet et al. (2011) with the star 2MASS J10185560-5856459. There is also an X-ray pulsar, PSR J1016-5857, on the edge or just outside the remnant (Camilo et al. 2001). Like HESS J0632+057, the high energy emission is believed to be due to interactions between a pulsar and the stellar wind (see the review by Dubus 2013) and both Waisberg \& Romani (2015) and Strader et al. (2015) favor a neutron star as the compact companion. We adopt the position and size of the SNR from Green (2019). Ruiz \& May (1986) estimate an age of $10^{4}$ years.

\section{A10 G296.5 +10.0}

G296.5+10.0 is associated with the X-ray PSR J1210-5226 (Zavlin et al. 2000). Halpern \& Gotthelf (2015) measure a proper motion of $(-12 \pm 5,9 \pm 8)$ mas/year. Giacani et al. (2000) estimate a distance of $2.1_{-0.8}^{+1.8} \mathrm{kpc}$, so we adopt a "parallax" of $\varpi=0.50 \pm 0.25$. We adopt the center and diameter (76') from Green (2019). Roger et al. (1988) estimate an age of 7000 year with uncertainties of a factor of 3 . de Luca et al. (2004) find $N(H)=(1.3 \pm 0.1) \times 10^{21} \mathrm{~cm}^{2}$, corresponding to $E(B-V) \simeq 0.22 \pm 0.02$, so for a distance of $4 \mathrm{kpc}$ we can probe down to $>2 M_{\odot}$.

\section{DATA AVAILABILITY STATEMENT}

All data used in this paper is publicly available.

\section{REFERENCES}

Abbott, R., Abbott, T. D., Abraham, S., et al. 2021, ApJL, 913, L7

Anderson, S. B., Cadwell, B. J., Jacoby, B. A., et al. 1996, ApJL, 468, L55

Aschenbach, B., Egger, R., \& Trümper, J. 1995, Nature, 373,587
Belczynski, K., Kalogera, V., Rasio, F. A., et al. 2008, ApJS, 174, 223

Benvenuto, O. G., Bersten, M. C., \& Nomoto, K. 2013, ApJ, 762, 74

Bietenholz, M. F. 2006, ApJ, 645, 1180

Bietenholz, M. F., Kondratiev, V., Ransom, S., et al. 2013, MNRAS, 431, 2590

Blundell, K. M., \& Bowler, M. G. 2004, ApJL, 616, L159

Boubert, D., Fraser, M., Evans, N. W., Green, D. A., \& Izzard, R. G. 2017, AAP, 606, A14

Bovy, J., Rix, H.-W., Green, G. M., et al. 2016, ApJ, 818, 130

Camilo, F., Bell, J. F., Manchester, R. N., et al. 2001, ApJL, 557, L51

Camilo, F., Stairs, I. H., Lorimer, D. R., et al. 2002, ApJL, 571, L41

Castelli, F., \& Kurucz, R. L. 2003, Modelling of Stellar Atmospheres, 210, 20P

Cha, A. N., Sembach, K. R., \& Danks, A. C. 1999, ApJL, 515, L25.

Chatterjee, S., Brisken, W. F., Vlemmings, W. H. T., et al. 2009, ApJ, 698, 250

Cigan, P., Matsuura, M., Gomez, H. L., et al. 2019, ApJ, 886,51

Clark, D. H., \& Stephenson, F. R. 1977, Oxford [Eng.] ; New York : Pergamon Press, 1977. 1st ed.

Corbet, R. H. D., Cheung, C. C., Kerr, M., et al. 2011, The Astronomer's Telegram, 3221,

Cutri, R. M. \& et al. 2012, VizieR Online Data Catalog, II $/ 311$

de Luca, A., Mereghetti, S., Caraveo, P. A., et al. 2004, AAP, 418, 625

Dinçel, B., Neuhäuser, R., Yerli, S. K., et al. 2015, MNRAS, 448, 3196

Dodson, R., Legge, D., Reynolds, J. E., \& McCulloch, P. M. 2003, ApJ, 596, 1137

Drimmel, R., Cabrera-Lavers, A., \& López-Corredoira, M. 2003, AAP, 409, 205

Dubus, G. 2013, A\&ARv, 21, 64

Eggleton, P. P. 1983, ApJ, 268, 368

Eldridge, J. J., Fraser, M., Smartt, S. J., et al. 2013, MNRAS, 436, 774

Eldridge, J. J., Stanway, E. R., Xiao, L., et al. 2017, PASA,

Fraser, M., \& Boubert, D. 2019, ApJ, 871, 92

Fruchter, A. S., Taylor, J. H., Backer, D. C., Clifton, T. R., \& Foster, R. S. 1988, Nature, 331, 53

Gaia Collaboration, Prusti, T., de Bruijne, J. H. J., et al. 2016, AAP, 595, A1

Gaia Collaboration, Brown, A. G. A., Vallenari, A., et al. 2021, AAP, 649, A1

Giacani, E. B., Dubner, G. M., Green, A. J., et al. 2000, AJ, 119, 281

Gotthelf, E. V., Helfand, D. J., \& Newburgh, L. 2007, ApJ, 654, 267

Gotthelf, E. V., \& Halpern, J. P. 2009, ApJL, 695, L35

Greco, E., Miceli, M., Orlando, S., et al. 2021, ApJL, 908, L45.

Green, D. A. 2014, Bulletin of the Astronomical Society of India, 42, 47

Green, G. M., Schlafly, E., Zucker, C., et al. 2019, ApJ, 887, 93

Green, D. A. 2019, Journal of Astrophysics and Astronomy, 
40, 36.

Gregory, P. C., \& Fahlman, G. G. 1980, Nature, 287, 805

Groh, J. H., Meynet, G., Georgy, C., et al. 2013, AAP, 558, A131

Guseinov, O. H., Ankay, A., \& Tagieva, S. O. 2005, Astrophysics, 48, 330

Gustafsson, B., Edvardsson, B., Eriksson, K., et al. 2008, AAP, 486, 951

Halpern, J. P. \& Gotthelf, E. V. 2015, ApJ, 812, 61.

Hamers, A. S., Rantala, A., Neunteufel, P., et al. 2021, MNRAS, 502, 4479

Han, Z.-W., Ge, H.-W., Chen, X.-F., et al. 2020, Research in Astronomy and Astrophysics, 20, 161

Henden, A. A., Templeton, M., Terrell, D., et al. 201

Hillwig, T. C., \& Gies, D. R. 2008, ApJL, 676, L37

Hinton, J. A., Skilton, J. L., Funk, S., et al. 2009, ApJL, 690, L101

Hobbs, G., Lorimer, D. R., Lyne, A. G., \& Kramer, M. 2005, MNRAS, 360, 974

Hoffleit, D. \& Warren, W. H. 1995, VizieR Online Data Catalog, V/50x

Hohle, M. M., Neuhäuser, R., \& Schutz, B. F. 2010, Astronomische Nachrichten, 331, 349

Hui, C. Y., \& Becker, W. 2006, AAP, 454, 543

Israelian, G., Rebolo, R., Basri, G., et al. 1999, Nature, 401, 142.

Jayasinghe, T., Stanek, K. Z., Thompson, T. A., et al. 2021, MNRAS, 504, 2577

Kerzendorf, W. E., Do, T., de Mink, S. E., et al. 2019, AAP, 623, A34

Kobulnicky, H. A., \& Fryer, C. L. 2007, ApJ, 670, 747

Kochanek, C. S. 2014, ApJ, 785, 28.

Kochanek, C. S. 2018, MNRAS, 473, 1633

Kochanek, C. S., Auchettl, K., \& Belczynski, K. 2019, MNRAS, 485, 5394

Koo, B.-C., Reach, W. T., Heiles, C., Fesen, R. A., \& Shull, J. M. 1990, ApJ, 364, 178

Koo, B.-C., Yun, M.-S., Ho, P. T. P., \& Lee, Y. 1993, ApJ, 417, 196

Kothes, R., Fedotov, K., Foster, T. J., \& Uyanıker, B. 2006, AAP, 457, 1081

Kothes, R., \& Foster, T. 2012, ApJL, 746, L4

Kramer, M., Lyne, A. G., Hobbs, G., et al. 2003, ApJL, 593, L31

Krause, O., Birkmann, S. M., Usuda, T., et al. 2008, Science, 320, 1195

Kulkarni, S. R., Clifton, T. C., Backer, D. C., Foster, R. S., \& Fruchter, A. S. 1988, Nature, 331, 50

Lam, C. Y., Lu, J. R., Hosek, M. W., et al. 2020, ApJ, 889, 31

Large, M. I., Vaughan, A. E., \& Mills, B. Y. 1968, Nature, 220,340

Leahy, D. A., \& Ranasinghe, S. 2012, MNRAS, 423, 718

Lewandowski, W., Wolszczan, A., Feiler, G., Konacki, M., \& Soltysiński, T. 2004, ApJ, 600, 905

Livingstone, M. A., Ransom, S. M., Camilo, F., et al. 2009, ApJ, 706, 1163

Lux, O., Neuhäuser, R., Mugrauer, M., et al. 2021, Astronomische Nachrichten, 342, 553.

Margon, B. 1984, ARA\&A, 22, 507

Marshall, D. J., Robin, A. C., Reylé, C., et al. 2006, AAP, 453,635
Marshall, H. L., Canizares, C. R., Hillwig, T., et al. 2013, ApJ, 775, 75

Mayer, M. G. F. \& Becker, W. 2021, arXiv:2106.00700

Michalik, D., Lindegren, L., \& Hobbs, D. 2015, AAP, 574, A115

Moe, M., \& Di Stefano, R. 2017, ApJS, 230, 15

Murray, S. S., Slane, P. O., Seward, F. D., Ransom, S. M., \& Gaensler, B. M. 2002, ApJ, 568, 226

Ng, C.-Y., Romani, R. W., Brisken, W. F., Chatterjee, S., \& Kramer, M. 2007, ApJ, 654, 487

Ng, C.-Y. \& Romani, R. W. 2007, ApJ, 660, 1357.

Ogata, M., Hirai, R., \& Hijikawa, K. 2021, MNRAS, 505, 2485.

Orosz, J. A., Kuulkers, E., van der Klis, M., et al. 2001, ApJ, 555, 489.

Panferov, A. A. 2017, AAP, 599, A77

Pastorelli, G., Marigo, P., Girardi, L., et al. 2020, MNRAS, 498, 3283

Patel, S. K., Kouveliotou, C., Woods, P. M., et al. 2001, ApJL, 563, L45

Pavlov, G. G., Zavlin, V. E., Sanwal, D., Burwitz, V., \& Garmire, G. P. 2001a, ApJL, 552, L129

Pavlov, G. G., Sanwal, D., Kızıltan, B., \& Garmire, G. P. 2001b, ApJL, 559, L131

Pejcha, O. \& Thompson, T. A. 2015, ApJ, 801, 90

Perryman, M. A. C., Lindegren, L., Kovalevsky, J., et al. 1997, AAP, 500, 501

Podsiadlowski, P. 1992, PASP, 104, 717

Kameswara Rao, N., Lambert, D. L., Reddy, A. B. S., et al. 2020, MNRAS, 493, 497.

Renzo, M., Zapartas, E., de Mink, S. E., et al. 2019, AAP, 624, A66

Rest, A., Foley, R. J., Sinnott, B., et al. 2011, ApJ, 732, 3

Reynolds, S. P., \& Aller, H. D. 1988, ApJ, 327, 845

Reynoso, E. M., Green, A. J., Johnston, S., et al. 2003, MNRAS, 345, 671

Reynoso, E. M., Cichowolski, S., \& Walsh, A. J. 2017, MNRAS, 464, 3029

Roberts, D. A., Goss, W. M., Kalberla, P. M. W., Herbstmeier, U., \& Schwarz, U. J. 1993, AAP, 274, 427

Roger, R. S., Milne, D. K., Kesteven, M. J., et al. 1988, ApJ, 332, 940.

Ruiz, M. T. \& May, J. 1986, ApJ, 309, 667.

Sana, H., de Mink, S. E., de Koter, A., et al. 2012, Science, 337,444

Sánchez-Cruces, M., Rosado, M., Fuentes-Carrera, I., \& Ambrocio-Cruz, P. 2018, MNRAS, 473, 1705

Skrutskie, M. F., Cutri, R. M., Stiening, R., et al. 2006, AJ, 131, 1163

Slane, P., Helfand, D. J., van der Swaluw, E., \& Murray, S. S. 2004, ApJ, 616, 403

Smith, N., Li, W., Filippenko, A. V., et al. 2011, MNRAS, 412, 1522

Sofue, Y., Furst, E., \& Hirth, W. 1980, PASJ, 32, 1

Strader, J., Chomiuk, L., Cheung, C. C., Salinas, R., \& Peacock, M. 2015, ApJL, 813, L26

Sukhbold, T., Ertl, T., Woosley, S. E., et al. 2016, ApJ, 821,38

Taylor, J. H., Manchester, R. N., \& Lyne, A. G. 1993, ApJS, 88, 529

Tendulkar, S. P., Cameron, P. B., \& Kulkarni, S. R. 2013, ApJ, 772, 31 
Thompson, G. I., Nandy, K., Jamar, C., et al. 1978,

Thompson, T. A., Kochanek, C. S., Stanek, K. Z., et al. 2019, Science, 366, 637

Toonen, S., Portegies Zwart, S., Hamers, A. S., et al. 2020, AAP, 640, A16

van den Bergh, S. 1980, Journal of Astrophysics and Astronomy, 1,67

van Leeuwen, F. 2007, AAP, 474, 653

Verbiest, J. P. W., Weisberg, J. M., Chael, A. A., Lee, K. J., \& Lorimer, D. R. 2012, ApJ, 755, 39

Villaseñor, J. I., Taylor, W. D., Evans, C. J., et al. 2021, arXiv:2107.10170

Waisberg, I. R., \& Romani, R. W. 2015, ApJ, 805, 18

Welsh, B. Y., Sfeir, D. M., Sallmen, S., \& Lallement, R. 2001, AAP, 372, 516

Wenger, M., Ochsenbein, F., Egret, D., et al. 2000, A\&AS, 143,9

Williamon, R. M., Dale, H., Evavold, C. L., et al. 2016, PASP, 128, 124202.

Winkler, P. F., Tuttle, J. H., Kirshner, R. P., \& Irwin, M. J. 1988, IAU Colloq. 101: Supernova Remnants and the Interstellar Medium, 65

Winkler, P. F., Long, K. S., Hamilton, A. J. S., et al. 2005, ApJ, 624, 189

Yao, J., Zhu, W., Manchester, R. N., et al. 2021, Nature Astronomy

Zacharias, N., Monet, D. G., Levine, S. E., et al. 2005, VizieR Online Data Catalog, I/297

Zavlin, V. E., Pavlov, G. G., Sanwal, D., \& Trümper, J. 2000, ApJL, 540, L25

Zeiger, B. R., Brisken, W. F., Chatterjee, S., \& Goss, W. M. 2008, ApJ, 674, 271 